\title{
„mit und in seiner Umwelt geboren"
}

\section{Frederik Buytendijks experimentelle Konzeptualisierung einer Tier-Umwelt-Einheit}

\author{
Julia Gruevska
}

\begin{abstract}
„being born with and in its environment". Frederik Buytendijk's Experimental Conceptualization of an AnimalEnvironment Unit
\end{abstract}

\begin{abstract}
The Dutch animal psychologist Frederik J. J. Buytendijk (1887-1974) developed an anti-reductionist approach in his ethological research of the 1920s and 1930s distinct from behaviorism, explicitly including in his experimental practices the freedom of animals, variable observations, and the subjective experience of the investigator. Buytendijk thereby developed a scientific theory that methodologically relied on phenomenology, hermeneutics, and concepts of unity based on gestalt theory, but did not abandon quantitative data collection. On the contrary, in his Groningen institute Buytendijk based his work on the biological theories of Jakob von Uexküll and specifically investigated the thesis of an "animal-environment unit". Using this institutional framework and two experiments (1924 \& 1931), this article determines to what extent Buytendijk was able to verify his statement that the animal was "born with and in its environment", which at the same time supported his scientificphilosophical concept. Accordingly, Buytendijk understood the environment as an organ of the animal, not only as a metaphor, but also as reality.
\end{abstract}

Keywords: Animal Psychology, Physiology, Behavioral Psychology, Environment, Philosophical Anthropology

Der niederländische Tierpsychologe Frederik J. J. Buytendijk (1887-1974) entwickelte in seinen Forschungen der 1920er und 1930er Jahre in Abgrenzung zum Behaviorismus eine antireduktionistische Zugangsweise auf Verhaltensexperimente. So bezog er in seinen Experimentalpraktiken explizit die subjektive Erfahrung des Versuchsleiters mit ein. Damit entwarf Buytendijk eine Wissenschaftstheorie, die methodologisch auf die Phänomenologie, Hermeneutik wie auf gestalttheoretische Ganzheitskonzepte zurückgriff, quantitative Datenerhebungen aber dennoch nicht aufgab. Vielmehr untersuchte Buytendijk auf der Grundlage des Biotheoretikers Jakob von Uexküll (1864-1944) in seinem physiologischen Institut in Groningen konkret die These einer "TierUmwelt-Einheit". Der vorliegende Beitrag ermittelt anhand der institutionellen Rahmenbedingungen und zweier Experimente (1924 und 1931), inwiefern Buytendijk seine Aussage, dass das Tier „mit und in seiner Umwelt geboren [ist]", die zugleich sein wissenschaftsphilosophisches Konzept stützte, zu verifizieren wusste. Dass die Umwelt dem Tier ein Organ ist, ist bei Buytendijk dementsprechend nicht nur metaphorisch, sondern ganz real zu verstehen. 
Tier-Umwelt-Konzepte im biophilosophischen Kontext der 1920er Jahre

Tiere dienen dem Menschen seit jeher als Objekte von Untersuchungen und Beobachtungen, die physiologisches wie psychologisches Wissen produzieren und Erkenntnisse zur lebendigen Wirklichkeit, nicht zuletzt über den Menschen selbst, liefern sollen. Spätestens seit Darwins Errungenschaften im Bereich der Deszendenztheorie gewannen Tierexperimente mit dieser epistemologischen Zielsetzung entscheidend an Konjunktur. Das lag auch daran, dass mit der biologischen Gleichstellung des Menschen mit dem Tier ein Transfer des Wissens über das Tier auf den Menschen unproblematisch erschien. Zurückführen lässt sich dies auf die Annahme eines „Mechanismus“, der besagt, dass unter denselben gegebenen Bedingungen die biologischen Prozesse von Körpern gleichartig verlaufen, so auch die Anpassung des Organismus an sein Lebensmilieu. Doch obwohl in der biologischen Forschung die Evolutionstheorie und ihr Paradigma, dass der tierische Organismus an seine Umwelt angepasst sei, zum state of the art avancierten, entwickelten sich aus einer vor allem deutschsprachigen philosophischen Denktradition konträre Positionen zum Mechanismusdenken der darwinschen Evolutionstheorie (vgl. unter anderem Lenoir 1982).

Der vorliegende Beitrag soll einen solch anders gelagerten Ansatz innerhalb der Umwelttheorien diskutieren, der zu Beginn des 20. Jahrhunderts in der Tierphysiologie und -psychologie auf dem europäischen Festland virulent wurde. Der vorzustellende Ansatz ist besonders bezeichnend, da er die Umwelt - und hier vor allem in der Interdependenz mit dem tierischen Leib - nicht als „gegebene Außenwelt" betrachtet, sondern sie als durch die Wahrnehmung der jeweiligen Lebewesen konstruiert auffasst. Dabei rückt der niederländische Tierphysiologe Frederik J. J. Buytendijk (1887-1974) in den Fokus der Untersuchung. Denn Buytendijk versuchte auf konkrete Weise im Rückgriff auf Jakob von Uexkülls Umweltkonzept und in konkreter Zusammenarbeit mit deutschen Philosophen, hauptsächlich Helmuth Plessner (1892-1985), Ganzheitskonzepte und Methoden der Phänomenologie und Hermeneutik in der Tierpsychologie zu etablieren (vgl. u.a. Becker 2015; Bühler 2004; Burgat \& Sommer 2016). Hierbei ging er von einem unabdingbaren Zusammenhang zwischen tierischem Leib und Umwelt aus, der durch seine dynamische und perzeptive Wechselwirkung als Organismus des Tiers bezeichnet werden könne. In diesem Sinne traf Buytendijk 1929 die prägnante Aussage: „[Das Tier] ist mit und in seiner Umwelt geboren“ (Buytendijk 1958 [1929]: 38). Diese zunächst rein theoretisch wirkende Hypothese bildet zum einen seine philosophische Auffassung von Mensch und Tier, die Buytendijk in die Riege der philosophischen Anthropologen des frühen 20. Jahrhunderts stellt und zum 
anderen seine experimentalpraktische Methodenkritik, die sich dem zu dieser Zeit stark etablierenden Behaviorismus entgegenstellt (vgl. u.a. Fischer 2009; van Buuren 2018; Gruevska 2019b). Diese Kritik (die vor allem in einem kantischen Sinn zu verstehen ist) kulminiert in einer spezifischen Wissenschaftstheorie Buytendijks, die eine Subjektinklusion sowohl beim Untersuchungsgegenstand als auch beim Untersuchenden in den Lebenswissenschaften notwendig macht. Die Berücksichtigung des Subjektiven ist für die Konzeption von Buytendijks Methode deswegen zentral, da er in seiner verstehenden Vorgehensweise im Experimentalsystem eine philosophisch-anthropologische Sicht auf Lebewesen als psycho-physisch konstituierte Einheiten, in der sich die Psyche durch das Physische ausdrückt, proklamiert (vgl. Gruevska 2018). Ausgehend von seinen Tierexperimenten, zunächst in Amsterdam (1919-1925), dann in Groningen (1925-1946), konstatierte Buytendijk, dass sich die Methoden der Phänomenologie und Hermeneutik zum Teil als geeigneter erwiesen als die in der Tierforschung üblichen quantitativen Datenerhebungen und vivisektorischen Eingriffe. Vor allem seien sie aber als Ergänzung der naturwissenschaftlichen Vorgehensweisen notwendig (Spiegelberg 1972). Diese Kombination von Analyse und Reflexion ermöglichte es ihm auch einer Kritik zu entgehen, die seine Position hätte als „unwissenschaftlich“ kennzeichnen können. Denn seine Thesen und Argumente belegte Buytendijk anhand seiner Experimente, Datenerhebungen und darüberhinausgehenden Beobachtungen, die sich sowohl auf Versuchstiere als auch seine eigene Vorgehensweise richteten. Dennoch, so muss eingestanden werden, schadete Buytendijks Anspruch seiner Reputation, weil seine Beiträge zur Tierpsychologie von Kollegen wegen seiner epistemologisch kritischen Implikationen als „unnecessary philosophical issues" weitgehend ignoriert wurden (Thines \& Zayan 1975: 88).

In der vorliegenden Studie soll anhand von zwei Experimenten (einem Labyrinthversuch mit Ratten und einer Formwahrnehmungsstudie mit Hunden) untersucht werden, wie Buytendijk seine Wissenschaftsphilosophie sowie These von einer Tier-Umwelt-Einheit begründete. In diesen Experimenten zeigt sich, wie konkrete Forschungspraktiken Begrifflichkeiten wie „Umwelt“ und „Tierwerdung“ konstituieren und gleichzeitig theoretische (philosophisch-anthropologische) Konzepte auf die Experimentalpraxis rückwirken (Rheinberger 2008: 1). Nicht nur theoretische Begriffe, sondern ebenso deren „materiale Einbindung in Praktiken und nichtdiskursives Wissen" werden daher im Folgenden betrachtet (Müller \& Schmieder 2008: XVII).

Ihren Ausgang nimmt die Studie aus einer allgemeinen Verortung des Umwelt-, aber auch Organismusbegriffs sowie einer daran anschließenden Spezifizierung des sich seit dem frühen 20. Jahrhunderts in der Biologie 
etablierten Begriffs „Umwelt“ Baron Jakob von Uexkülls. Vor diesem Hintergrund soll eine Kontextualisierung Buytendijks biologischer Sichtweise vorgenommen werden. Uexküll, der als ein wichtiger Referenzpunkt in der deutschsprachigen Philosophie phänomenologisch-hermeneutischer Tradition fungiert, bildet dementsprechend auch für Buytendijk den Anlehnungspunkt, an dem die Theorie der Tier-Umwelt-Einheit, die Kritik am Behaviorismus und Buytendijks eigene spezifische Wissenschaftsphilosophie ansetzt. Daran anschließend soll Buytendijks sowohl institutioneller als auch ideeller Bezugsrahmen vorgestellt werden, um seine wissenschaftliche Position nicht nur als Theoretiker, dem eine metaphysische Kritik angelastet werden kann, sondern auch als Praktiker, der seine Philosophie im Abgleich und aus der experimentellen Arbeitsweise er- und begründete, $\mathrm{zu}$ verdeutlichen. Mit einer solchen systematischen Aufarbeitung der verhaltens- und wahrnehmungspsychologischen Experimente Buytendijks soll beispielhaft ein Blick auf seine vielversprechende Bio- und Umwelttheorie sowie ihre implizite „philosophische Durchdenkung“ (Buytendijk 1927) geworfen werden, welche die Streitfrage nach einer geforderten Objektivität in den Lebenswissenschaften erneut entfachen und in ein neues Licht rücken kann.

\section{„Umwelt" und „Organismus" - eine begriffshistorische Skizze}

Wirft man einen Blick in die enzyklopädischen Einträge des Lemmas „Umwelt", so ist man häufig erstaunt über deren Umfang. Im Historischen Wörterbuch der Philosophie umfasst „Umwelt" sieben Spalten, im Historischen Wörterbuch der Biologie sogar 42 Seiten. Schaut man sich jedoch das Diskursfeld zum Begriff der „Umwelt“ an, scheint diese Menge an Material nicht mehr verwunderlich. Denn der Begriff „Umwelt“ erweist sich als ebenso vielseitig wie kontrovers, seine Definition in den verschiedenen Disziplinen ebenso statisch wie dynamisch, seine Tradition ebenso linear wie brüchig (vgl. u.a. Toepfer 2011). Seit der französische Naturwissenschaftler George Cuvier (1769-1832) für die Biologie den Terminus des „Milieus“ unter der Definition prägte, dass Organismen nur unter bestimmten äußeren Bedingungen existieren können, ging man auch von einem „inneren Milieu“ von Lebewesen aus, das sich aus den „Verhältnissen im Organismus" konstituiert (Potthast 2010: 50). In Deutschland, wird im Historischen Wörterbuch der Philosophie festgestellt, sei der Begriff „Umwelt“ zu Beginn des 19. Jahrhunderts aufgekommen. Dort bündelte der Begriff „die Gesamtheit der Aspekte einer in quasi-natürlicher Unmittelbarkeit erfahrenen Umgebung“ (Müller 2001: 99), wobei die Umwelt des 
Menschen erst durch die Vermischung des in Frankreich bereits existierenden Begriffs des „Milieus“ als Terminus im Sinne eines sozialen, geistigen Raums Eingang fand. ${ }^{1}$ Unterdessen gewannen aber auch Abhandlungen und Studien durch die Erkenntnisse der Evolutionstheorie an Konjunktur, die sich mit „Lebens- und Organisationsgemeinschaften, über Abhängigkeiten und Wechselwirkungen in mehreren wissenschaftlichen Bereichen" beschäftigten (Müller 2001: 100). Jener Umweltbegriff, der sich seit Beginn des 20. Jahrhunderts, vor allem in der Biologie, aber auch alltagssprachlich als solcher etabliert hat, ist auch derselbe Umweltbegriff, der dem vorliegenden Beitrag als Semantik zugrunde liegt: Der Umweltbegriff, den Jakob von Uexküll 1907 einführte und der sich allgemein als „physiologische[] Wechselwirkungen von Organismen mit bestimmten Bereichen der Umgebung“ (Müller 2001: 100) versteht. Der Begriff des „Organismus“ ist im Gegensatz zum Umweltbegriff ungemein älter, obwohl er ebenfalls erst seit dem 18. Jahrhundert im allgemeinen wie fachlichen Sprachgebrauch genutzt wurde (Ballauff et al. 1984: 1330). In dieser langen Tradition hat der Begriff viele Wandlungen sowie Transfers in verschiedene Disziplinen und Denkmodelle erfahren. So wurde der Begriff "Organismus“ nicht nur in den Naturwissenschaften gebraucht, sondern unter anderem in philosophische, soziologische und psychologische sowie politische Diskurse als Fachterminus integriert (Ibid.: 1330-1358). Im Rahmen des hier vorgestellten Konzepts von Buytendijk, der explizit von lebenden Organismen in der physiologischen Forschung ausgeht, scheint es sinnvoll, sich an Georg Toepfers (2017) konzise Definition des Organismusbegriffs zu halten, die lautet wie folgt: „Lebendige Organismen sind ganzheitliche, funktional geschlossene Einheiten. Und lebendige Organismen sind in eine jeweilige Umwelt eingebettete, mit Objekten dieser Umwelt interagierende, offene und dynamische Systeme" (Toepfer 2017: 3). Entsprechend muss der Organismus auch bei Buytendijk auf diesen zwei Ebenen betrachtet werden: in der Interaktion der tierischen Innenwelt und Außenwelt (im engeren Sinne: mit seiner Umwelt ${ }^{2}$ ) sowie im daraus kulminierenden physiologischen Gesamtzusammenhangs von Tier und Umwelt.

In seinem aufschlussreichen Beitrag zur „Biologie und Anthropologie der Wahrnehmung" konstatiert Toepfer weiter, dass bei den Autoren des 20. Jahrhunderts, darunter explizit Buytendijk, die Vermittlung von Organismus und Umwelt die Wahrnehmung der Lebewesen übernahm. Den Konnex Organismus und Umwelt erklärt Toepfer als evolutionäres Resultat, weil „sowohl die Integrität der evolvierenden Systeme als funktional und morphologisch geschlossene Systeme aus interdependenten Komponenten (Organismen) als auch die feste Kopplung dieser Systeme an Objekte der Umwelt, die nicht Teil der strukturell-funktional geschlossenen Gestalt- und Herstellungseinheit von Organismen sind, mit denen zusam- 
men sie aber darüber hinaus ein übergeordnetes System bilden (Organismus und Umwelt) [stabilisiert wurde]" (Toepfer 2017: 3). Dieser Gedanke findet sich auch bei Uexküll und Buytendijk: Ein solches übergeordnetes System nannte Uexküll den „Bauplan“, der zur Umsetzung des „Funktionskreis[es]“ fungiert (Uexküll 1909: 7), und Buytendijk „Situation“ (Buytendijk \& Fischel 1931: 452). Für Buytendijk ist eine "Situation“ ein dynamisches, gleichursprüngliches Ereignis zwischen Tier und Umwelt, das eine Einheit darstellt und von Parametern wie Einsicht, Gewohnheit, Erfahrung und Verstehen (von Sachbezügen wie von Aufgaben) getragen wird (ibid.). Diese Art der Auffassung von Tier-Umwelt-Relationen war zu Beginn des 20. Jahrhunderts keine Seltenheit, verwunderlich ist eher, dass sich die Autoren wenig aufeinander bezogen haben. „Offenbar haben sie ihre Vorstellung meist unabhängig voneinander entwickelt" lautet Toepfers Vermutung dazu (2017: 12). Im Falle Buytendijks trifft diese Aussage allerdings nicht zu, weil er die Konzeptionen Uexkülls ausdrücklich in Theorie und Praxis aufnahm, reflektierte und weiterdachte. Die Auseinandersetzung mit Uexküll spielte zudem eine wichtige Rolle für Buytendijks philosophisch-anthropologische Ideen, die er in den frühen 1920er Jahren im Austausch mit dem Philosophen Helmuth Plessner generierte (Buytendijk \& Plessner 1925, 1980 [1935]). Plessners „Logik der lebendigen Formen“ (Plessner 1985: 327), die in vielerlei Hinsicht auf Buytendijks Arbeiten zurückgeht, hat der Philosoph Kristian Köchy treffend als „Erweiterung des Uexküll Programms" bezeichnet (Köchy 2015). Mittels einer Rezension der bis dahin veröffentlichten uexküllschen Werke im Vakblad foor Biologen (September 1921) versuchte Buytendijk Uexküll zudem auch in den Niederlanden bekannt und seine Theorie zugänglich zu machen.

In einem Brief an Buytendijk vom 8. November 1921 bedankte sich Uexküll bei dem Niederländer und merkte an, dass er beim nochmaligen Lesen Buytendijks Schriften keine Unterschiede zwischen ihren beiden Theorien feststellen konnte und, dass er gerne die "Gelegenheit gehabt hätte“, mit Buytendijk, „die Grundprobleme der Biologie durchzusprechen“. „[Besonders] dankbar" sei er aber, dass "so ein strenger Logiker" wie Buytendijk seiner "auf Anschauung gegründeten Ansichten, Beifall“ zolle, denn im Ganzen sei er „allen Logikern und Mathematikern ein Gräuel. Ganz zu

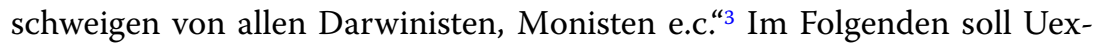
küll sowie seine für Buytendijk (auch im Lichte der philosophischen Anthropologie des 20. Jahrhunderts) bedeutungsvolle Umwelt-Theorie konzis skizziert werden. 


\section{Der Umweltbegriff bei Jakob von Uexküll}

Baron Jakob Johann von Uexküll bildet in der philosophischen Anthropologie und Naturphilosophie des 20. Jahrhunderts häufig den biotheoretischen Referenzpunkt. Prominent zu nennen sind in dieser Tradition die Philosophen Max Scheler (1874-1924), der bereits erwähnte Helmuth Plessner und in kulturanthropologischer Manier Ernst Cassirer (1874-1945). Für die Ausarbeitung der Beziehung von Organismus und Umwelt in Korrelation zur Mensch-Welt-Beziehung ist der Biotheoretiker für die philosophische Anthropologie, deren Untersuchungs- und Zielgegenstand der Mensch ist, auf zwei Ebenen interessant: Zunächst formal, denn Uexküll versuchte eine Grundlegung zu schaffen, an dem alle weiteren biologischen Theorien anknüpfen können (Block 2016). So geht es ihm in einem seiner Hauptwerke, „Umwelt und Innenwelt der Tiere“ (1909, 1921), nicht darum, einzelne Aspekte des tierischen Lebens oder des Organismus zu untersuchen, sondern diese als Beweismaterial zu nutzen, um seine Theorie des „Funktionskreises“ zu stützen. Im Grunde machte er es sich zur Aufgabe, der Spur des Lebendigen bis zum Basalsten zu folgen und dabei die natürlichen, sich immer wiederholenden Prozesse im Organismus offenzulegen. Damit wäre nicht nur eine Analogisierung von Organismus und Maschine gerechtfertigt, sondern ebenso der Physiologie eine Konstante an die Hand gegeben, wie mit dem bzw. am Tier geforscht werden könne. In einer ähnlichen Weise ist die Konstitution eines universellen Menschenbildes das Anliegen der philosophischen Anthropologie - neben der Herausstellung des Wesens des Menschen in epistemologischer Absicht. So sah es die philosophische Anthropologie als ihre Pflicht an, das Wesen des Menschen - das heißt, die Bedingung der Möglichkeiten der Ausbildung spezifisch menschlicher Eigenschaften - herauszukristallisieren und somit den Wissenschaften, die den Menschen zum Gegenstand haben, ein gemeinsames Fundament bereitzustellen (Plessner 1975 [1928]; 2019 [1961]; Scheler 2007 [1928]). Im Laufe der Philosophiegeschichte war die anthropologische Frage schon immer präsent: Bereits in der Antike findet man anthropologische Modelle (siehe Platons Dihairesis des Menschen als ungefiederter Zweibeiner), in der Frühen Neuzeit bei René Descartes (1596-1640), bei Immanuel Kant (1724-1804) findet man ihn sogar wortwörtlich in seiner „Anthropologie in pragmatischer Hinsicht“ (1796/97). Darin begrüßt Kant die Zusammenarbeit von Philosophie und Einzelwissenschaften, allerdings unter Ausschluss der für die Anthropologie des 20. Jahrhunderts so fundamentale Physiologie (vgl. Marquard 1971: 366). Selbst Friedrich Nietzsche (1844-1900) hat einen Begriff vom Menschen als "das noch nicht festgestellte Thier“ (Nietzsche 1980 [1886]: 81). In all diesen Modellen wird der Mensch als Tier mit einer distinktiven Besonderheit beschrieben, beispielsweise als animal 
rationale oder zoon politikon (vgl. Hartung 2008). Aber das Bezeichnende an der philosophischen Anthropologie des 20. Jahrhunderts ist, dass an der kategorialen Unterscheidung von Mensch und Tier angesetzt und vor diesem Hintergrund rekonstruiert wird, auf welche dem Menschen genuine Fähigkeit alle dem Menschen spezifischen Ausdrucksweisen zurückzuverfolgen sind. Eine solche These der anthropologischen Differenz ist in nachdarwinschen Zeiten nur „informativ“, wenn sie eine grundlegende Unterscheidung vorweisen kann, in die Evolutionstheorie eingebettet ist sowie einer empirischen Prüfung unterzogen werden kann (Wunsch 2016: 260). In Helmuth Plessners Biophilosophie, die an entscheidenden Stellen maßgeblich auf der Umwelttheorie Uexkülls basiert (Schmieg 2017; Köchy 2015), ist es die „exzentrische Positionalität“ des Menschen, die, bereits begrifflich angelegt, eine Beziehung des Menschen zur Welt aussagt. Als Kontrast, oder zumindest Andersgelagertheit zur Lokalisierung des Tiers als mit und in seiner (selbst konstruierten) Umwelt lebend, ist dieser Aspekt als strukturale Differenz angelegt. Die empirische Belegbarkeit wurde unter Einbezug Uexkülls praxisbezogener Biotheorie erfüllt und mit Buytendijk tatsächlich nach diesen Fragen ausgerichtet experimentell untersucht. Ähnlich wie Uexküll verfolgte Buytendijk die Aufgabe eine Biotheorie aufzustellen, die ausgehend von der Analyse des Tiers auch einen Transfer auf den Menschen schafft. Damit geht er über Uexkülls Bestreben hinaus, um die „Lebenserscheinungen“ auch zu „verstehen“, anstatt sie nur $\mathrm{zu}$ „erklären“.

Uexküll selbst überträgt in der Auseinandersetzung mit der „Umwelt und Innenwelt der Tiere" nicht - zumindest nicht ohne darauf hinzuweisen, dass es metaphorisch zu verstehen ist - seine Erkenntnisse vom Tier auf den Menschen. Er betont vielmehr, dass das Tier gerade nicht mit menschlichen Parametern bemessen werden darf, was vor allem aus der Subjekt-Umwelt-Abhängigkeit hervorgeht. Das bezeugt schon die Frage nach der Umwelt des Tiers und was als Umwelt gilt. Denn die tierische Umwelt ist ein Konstrukt, das sich aus der Wahrnehmung der individuellen Tiere selbst ergibt und nicht das, was der Mensch als „Umgebung“ des Tiers wahrnimmt, auch wenn problematisch bleiben muss, dass der Mensch als Beobachter in dieser Konstellation seinem eigenen menschlichen Horizont unterliegt (Uexküll 1909: 248).

Diese Umweltkonzeption Uexkülls war von Seiten der Biologie stark kritisiert worden, da sie sich als individualistische Biologie in letzter Konsequenz nicht als "theoretisch oder methodisch [...] strenge Naturwissenschaft" aufstellen könne (vgl. Mildenberger \& Herrmann 2014: 314). Prominent kritisierte Hermann Weber (1899-1956) Uexkülls Umweltmodell, in dem er es „per se als ungenügend ablehnte“ (ibid.) und ihm in seinem Aufsatz Zur Fassung und Gliederung eines allgemeinen biologischen 
Umweltbegriffs (1939) einen „objektiveren“ Umweltbegriff entgegenstellte. Doch gab es von Seiten der Gestaltpsychologie einige Befürworter, vor allem von denjenigen, die auch Buytendijks Forschungsprogramm verfolgten. So waren Adolf Mayer-Abich (1893-1971), Friedrich Alverdes (1889-1952) und Otto Koehler (1889-1974) Figuren, die das Umweltkonzept aufnahmen, aber auch für ihre Interessen weiterentwickelten (Mildenberger \& Herrmann 2014: 314).

Für Uexküll ist die „Welt, die uns umgibt, [...] die objektive Wirklichkeit, mit der wir es allein zu tun haben, wenn wir objektive Naturforschung treiben." (Uexküll 1909: 248) Mit den Tieren, die ebenfalls Bestandteil der menschlichen Wirklichkeit seien, verhalte es sich allerdings anders: Ihnen sei die Wirklichkeit nicht in dieser Fülle, wie wir sie wahrnehmen, gegeben. Jedes Tier besitze, je nach Art, Gattung und individuellem Exemplar seine eigene Umwelt, „die immer größere Verschiedenheiten mit der unseren aufweist, je weiter es sich in seiner Organisation von uns entfernt" (ibid.: 249). Uexküll schränkt so die tierische Umwelt ein, indem er zur Umwelt zugehörig bloß solche Phänomene zählt, die direkt auf den Körper des Tiers einwirken und es erregen. Das bedeute aber nicht, dass Umwelten statisch blieben, die Umwelten der Tiere könnten sich auch verändern, sobald neue Reize oder Reizquellen aufträten. Nur die Umgebung, die die „Umwelt“ des Menschen ausmacht, ändere sich nicht. Damit verdeutlicht Uexküll die Demarkation, die den Menschen und das Tier trennt und die uns nicht erlaubt, explizite Urteile über die Umwelt der Tiere zu fällen: „Während die Umwelt sich ändert, bleibt die Umgebung im wesentlichen unverändert, weil sie eben die Umwelt des Beobachters und nicht des Tieres darstellt" (ibid.). Unveränderlich bliebe im Reich der Tiere aber „[d]as Verhältnis zwischen Innenwelt und Umwelt" (ibid.), weshalb Uexküll dies zu einem universellen Prinzip erhebt.

Uexkülls Theorie soll anhand seiner Beobachtungen zur Amöbe (amoeba terricola) veranschaulicht werden. Die Umwelt der Amöbe, so führt Uexküll aus, ist nicht das, was die Amöbe an Gegenständen und Orten umgibt, also das, was der Mensch in seiner Beobachtung der Amöbe wahrnimmt, sondern vielmehr „hängt die Amöbe in ihrer Umwelt wie an dreierlei Arten von Gummifäden, die sie ringsum halten und alle ihre Bewegungen lenken und bestimmen. Dieser kleine Ausschnitt der Welt ist eine in sich zusammenhängende Welt, einfacher und widerspruchsloser als die unsere, aber ebenso planvoll, ebenso künstlerisch" (ibid.: 39). So bestimmt, rein physiologisch gesehen, bei einer Amöbe lediglich das Ektoplasma das, was als Umwelt der Amöbe „bezeichnet werden kann“ (ibid.: 37): „Die ganze biologische Aufgabe, die Wirkungen der Umwelt aufzunehmen und in entsprechende Bewegungen zu verwandeln, liegt dem Ektoplasma ob,“ 
denn „Tier und Umwelt bilden zusammen eine geschlossene Zweckmäßigkeit" (ibid.: 49).

Damit erweist Uexküll der ganzen Biologie einen Dienst, denn „[nur] wenn alle Hände nach einem gemeinsamen Plane tätig sind, um auf fester Grundlage ein Haus zu erbauen, kann etwas Gedeihliches und Dauerndes entstehen“ (ibid.: 2). Als Punkt, an dem man zusammenarbeiten müsste, setzt Uexküll an den „schönen Zeiten“ an, „da Anatomie und Physiologie noch ungetrennt eine einheitliche Biologie bildeten“, denn da "faßte man jedes Tier als funktionelle Einheit auf. Die anatomische Struktur und ihre physiologischen Leistungen wurden gleichzeitig erforscht und als zusammengehörig betrachtet" (ibid.: 3). Mit der Trennung der beiden Wissenschaften „traten tote räumliche Beziehungen an die Stelle der lebendigen Wechselwirkung der Organe“ (ibid.). Man nahm das Tier auch nicht mehr als "funktionelle Einheit" wahr, sondern studierte einseitig möglichst isolierte Teilfunktionen (ibid.: 4). Uexküll kritisiert die darwinistischen Strömungen und macht sie dafür verantwortlich, dass die Physiologie „vollkommen unterdrückt" wurde. Sie würden lediglich das Problem verfolgen, wie sich „die Struktur der höheren Tiere aus der der niederen entwickelt habe“ (ibid.). Die Annahme war, dass es eine ansteigende, stufenweise Vervollkommnung gäbe, bei der die einfachsten Strukturen die unterste Stufe bildeten und die komplexeren Strukturen die vollkommensten. Für Uexküll ist diese Annahme jedoch völlig fehlgeleitet: Denn „ein Pferd [würde] die Rolle eines Regenwurms nur sehr unvollkommen ausfüllen“ (ibid.). Das Problem, das der Darwinismus hier preisgäbe sei, dass er die Fähigkeiten und Strukturen der Tiere am Menschen messe, weshalb er zu diesem Fehlschluss der Vollkommenheitsthese gelange.

Uexküll wehrt sich auch gegen die Behauptung, das Lebewesen wäre an seine Umgebung vollkommen angepasst, vielmehr sieht er es umgekehrt, „der Bauplan schafft in weiten Grenzen selbsttätig die Umwelt des Tieres“ (ibid.: 5). Uexküll führt deshalb auch gegen den darwinschen Begriff der „Angepasstheit“, den Begriff der „Einpassung“ ins Feld; einen Begriff, den auch Buytendijk in seinen Schriften verwendet, wie noch gezeigt wird.

Damit gibt Uexküll auch eine Anleitung, wie die Theorie auf die Praxis des Naturwissenschaftlers übertragen werden müsse. Es sei die Aufgabe des Experimentators, festzustellen, „welche Teile d[er] Umgebung auf das Tier einwirken und in welcher Form das geschieht" - eine einfache Beobachtung reiche dafür nicht aus: „Unsere anthropozentrische Betrachtungsweise muß immer mehr zurücktreten und der Standpunkt des Tieres der allein ausschlaggebende werden“ - der Experimentator muss sich zurückziehen, gerade innerlich - so muss alles, was das Tier und unsere Fragestellung nicht betrifft, „verschwinden“ (ibid.: 6). Buytendijk hat einen ebensolchen Anspruch, verfolgt und begründet ihn aber vor allem durch 
seine Experimente, und bettet ihn in den philosophischen Diskurs um die Subjekt-Objekt-Dispute ein, die sich spätestens seit der Begründung der Phänomenologie wieder entfachten.

Was Uexküll hier für die Einzeller und wirbellosen Tiere, wie etwa das Paramaecium oder die Amöbe, annimmt, stellt Buytendijk auch als These für sowohl große als auch kleine Säugetiere unter differenzierteren Darstellungen auf. Schaut man sich die Position Buytendijks genauer an, so wird deutlich, dass hier eine Sensibilität für die Reflexion des Beobachtenden bzw. des Experimentators eingefordert wird: Ein Zusammendenken und voneinander Ableiten von Mensch und Tier wird zwar vorgenommen, aber auf einer anderen Ebene. In der (zeitlich) intensiven Beobachtung sowie Beschäftigung mit dem Tier wird es dem Experimentator möglich, Unterschiede und Gemeinsamkeiten zwischen Tier und Mensch festzustellen. Dabei ist es zwar richtig, aber vor allem wichtig, dass der Experimentator sich als Beobachtender begreift. Das experimentierende Subjekt führt, so Buytendijk, nicht mehr zu Verschleierung des Objektiven oder des Objekts, sondern avanciert zur Chance gerade das Objektive des individuellen Untersuchungsobjekts freizulegen (vgl. Gruevska 2018). Buytendijks Argumentation rührt zwar ähnlich wie bei Uexküll her von den Auseinandersetzungen mit den biotheoretischen Ansätzen seiner Zeit, den Entwicklungen in den Lebenswissenschaften sowie den Experimenten, doch geht er noch darüber hinaus, indem er seine Kritik und Theorie an philosophischen Diskussionen anlehnt und diese dort auch fruchtbar macht.

Mithilfe der subjektiven Beobachtung und der intensiven Beschäftigung mit den "Ausdruckserscheinungen des Lebendigen“ - unromantischer ausgedrückt: des Verhaltens der Versuchstiere - ist es dem Forscher möglich, sich in das Tier einzufühlen, es zu verstehen.

Möglich wird dies nur, so Buytendijk, wenn man sich ganz im goetheschen Sinn, dem Naturobjekt hingibt. Für einen Physiologen des 20. Jahrhunderts, der in der Tradition von Johannes Müller und Ernst Brücke über Wilhelm Kühne bis hin zu Hendrik Zwaardemaker steht, bedeutete diese Hingabe allerdings nicht, dass man die Tiere in ihrer natürlichen Umwelt, im Freiland beobachtete (wie etwa Konrad Lorenz ab den 1930er Jahren). Vielmehr bedeutete es, dass man die Tiere im Labor auf viele verschiedene Weise untersuchte und mit Blick auf den Primaten einer Tier-UmweltEinheit die Versuchsanordnung für das Tier so natürlich wie möglich gewissermaßen „naturimitierend“ - gestaltete (vgl. Gruevska 2019a). Ganz explizit betont Buytendijk die Vorteile eines solchen Natürlichkeit suggerierenden Labors gegenüber den beiden anderen Extremen, dem behavioristischen Labor und der Beobachtung im Freiland. Auf das behavioristische Labor wird im weiteren Verlauf des vorliegenden Aufsatzes dezidiert in der Aushandlung mit Buytendijks Experimenten eingegangen. Bei der 
Beobachtung im Freiland kann konzis darauf hingedeutet werden, dass Buytendijk es für unabdingbar und notwendig hält, die Kontrolle über die etwaigen dem Tier gestellten Aufgaben sowie die Gegebenheiten der hier angeordneten „Umwelt" des Tiers zu behalten. ${ }^{4}$

Um einen Einblick in diese spezifische praktische Arbeit Buytendijks zu geben, soll zunächst sein physiologisches Institut in Groningen vorgestellt werden, um zu verdeutlichen in welcher Größenordnung er seine Experimente durchgeführt hat. Dabei rücken die Fragestellungen in den Fokus, unter welchen epistemischen wie institutionellen, theoretischen wie materiellen Bedingungen und unter welchen Verfahren Tiere „zu Objekten des Wissens“ gemacht wurden (Rheinberger 2007: 11). Besonders interessant ist dabei, dass Buytendijk häufig mit technischen Apparaturen arbeitete, die er allerdings mit der von ihm präferierten Methode der Hermeneutik und Phänomenologie auswertete, statt wie zu seiner Zeit üblich in physikalistischer, reduktionistischer Manier.

\section{Das physiologische Institut der Rijksuniversiteit Groningen}

Das physiologische Institut der Rijksuniversiteit Groningen, an dem Buytendijk ab 1925 als Leiter fungierte, wurde im Jahre 1911 eröffnet und ersetzte damit das bereits seit 1866 existierende Vorläuferinstitut. Das Institut in Groningen war das erste in den Niederlanden, das sich ausschließlich als physiologisches Institut verstand. Seit Beginn des 19. Jahrhunderts entwickelte sich die Physiologie samt ihrer Methoden und Techniken so rasant, dass das ursprüngliche Institut Anfang des 20. Jahrhunderts längst nicht mehr mit den Anforderungen der Wissenschaft mithalten konnte. Hartog Jakob Hamburger (1859-1924), der das Groninger Institut seit 1901 leitete, gelang es, staatliche Gelder einzuwerben und ein neues Institut zu errichten, das durch neueste technische Apparate und zahlreiches Equipment international zu einer der vorbildlichsten und einflussreichsten Institutionen für die physiologischen Labore der Zeit wurde. Das neue physiologische Institut wurde am 7. April 1911 in Groningen eröffnet (de Haan 1925: 35f.). Buytendijk setzte die Aufgabe Hamburgers nach dessen Tod fort, jedoch mit anderen Methoden und unter anderen ideellen Vorzeichen, wie nach einer kurzen Übersicht über das Institut im weiteren Verlauf deutlich werden soll.

Schaut man auf die Lage sowie Anordnung des physiologischen Institutes, so befand es sich auf einem 1,3 ha großen Grundstück der Universität Groningen, das neben einem Hauptgebäude auch mehrere Ställe, Tiergehege, Weiden und Gemüsebeete beherbergte (s. Abb. 1). Daneben gab 


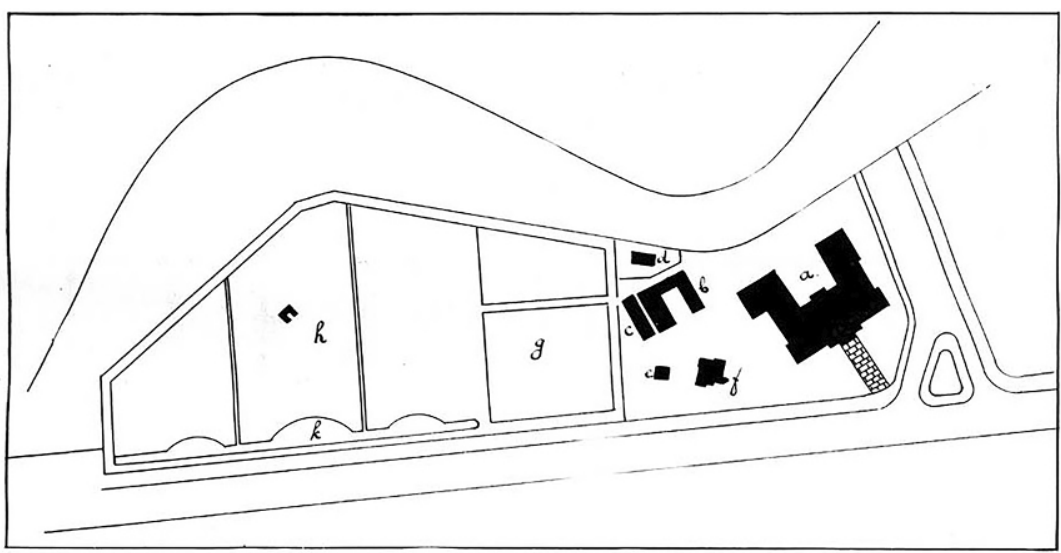

FIG. 3

$\begin{array}{ll}\text { a. Main building } & \text { f. House for custodian } \\ \text { b. Stable } & \text { g. Greens and clover } \\ \text { c. Storage } & \text { h. Pasture } \\ \text { d. Rabbits } & \text { k. Pond } \\ \text { e. Glass room } & \end{array}$

Abb. 1 Anlage des Groninger Institutes mit Gärten, de Haan: University of Groningen: Physiological Institute: 37

es auch zahlreiche Teiche und Aquarien, wodurch das Labor über eine konstante Versorgung mit Wasserflora und -fauna im natürlichen Zustand verfügte (de Haan 1925: 41). Das Hauptgebäude bestand aus drei Gebäudeteilen, die sich über drei Etagen verteilten, in dem sowohl die Seminare und Vorlesungen für die Studenten - überwiegend in Medizin - als auch die Untersuchungen und Experimente mit den Tieren stattfanden (ibid.: 37). Neben den verschiedenen Laboren (Histologie, Bakteriologie, Elektrophysiologie) umfasste es Unterrichtsräume, Vorlesungssäle, Vorführräume sowie Abstell- und Reparaturräume für die verschiedenen für die Experimente genutzten Apparaturen. Der Seminarraum war mit einem Kinematographen ausgestattet, der häufig zum Einsatz kam und nicht bloß die Aufnahmen von (internationalen) Kolleg*innen zeigte, sondern mit dem auch eigene Experimente und Untersuchungen aufgezeichnet wurden. Die Vorteile eines solchen Kinematographen waren immens, denn nicht nur dienten sie der Konservierung und dem nachträglichen Nachvollzug von Experimenten, sondern sie ersparten auch viel Zeit und Material - und nicht zuletzt auch den Versuchstieren den Tod. Auf derselben Ebene wie die Unterrichtsräume befand sich die Bibliothek sowie der sogenannte $b a$ lance room, ein Raum, in dem eine kleine Bücherauswahl, Zeitschriften, Broschüren und Zeitungen zugänglich waren, die dem Direktor gehörten. Buytendijks Mitarbeiter schon aus Amsterdamer Zeiten, Johann Bierens de Haan, kommentierte diesen ungewöhnlichen Raum damit, dass die Bücher 
den Studenten, Doktoranden und Assistenten zur allgemeinen Nutzung zur Verfügung gestellt werden sollten: „By keeping them here they can be made of wider use than they would be in the professor's home" (ibid.). Es gab vier Labore für die allgemeinen Forschungen: eins für die wissenschaftlichen Mitarbeiter und eins für die Doktoranden; ein weiteres kleines Labor, das als das assistants' laboratory bekannt war sowie das private Labor des Direktors Buytendijk. All diese Labore waren mit Zentrifugen, Luftdruckund Absauggeräten sowie Heizöfen für die Temperaturregulierung ausgestattet. Im Erdgeschoss befand sich zudem das physiologische Labor für die Studenten und ein weiteres für physiologische Chemie sowie dazwischen ein darkroom für die spektroskopische Untersuchung von Blut, der Ophthalmoskopie (Augenspiegelung), aber auch zur Erforschung der Tiere mit Röntgenstrahlen, für die vollständige Dunkelheit unerlässlich war. Der enorme Fortschritt in der Röntgentechnik ermöglichte es, die Bewegungen von Darm, Herz, Lunge und anderen Organen vor und nach Operationen kinematographisch zu untersuchen und einer Analyse zu unterziehen. Die für die Ausstattung dieses Raumes notwendigen Mittel waren bis zum Direktionsantritts Buytendijks nicht verfügbar. Erst Buytendijk führte diese Innovationen im physiologischen Institut ein, indem er die finanziellen Mittel dafür aufbrachte (ibid.: 41).

Das physiologische Institut in Groningen hatte zudem einen Schwerpunkt auf der Untersuchung größerer Säugetiere, wie zum Beispiel Pferde, Kühe, Ziege oder Hunde, weshalb es großräumigere Labore und umfangreichere Räume zur Geräteaufbewahrung gab (verschiedene Zentrifugen, große Installationen zur Ultrafiltration, eine Bucher Presse zur Herstellung von Säften, ein Destillationsapparat, Maschinen zur allgemeinen Luftabsaugung und Druckausübung sowie Temperaturregler und ein String Galvanometer) (ibid.: 38; s. Abb. 2). In den Laboren gab es dazu noch spezielle Apparaturen zum Festschnallen der Versuchstiere, sodass die Sicherheit von Operateur und Tier gewährleistet werden konnte. Das Arbeiten am lebendigen Organismus bedurfte in der Vorbereitung und in der Nachbereitung viel Zeit. So war das physiologische Labor in Groningen so ausgestattet, dass so wenig Zeit wie möglich für diese (eigentlich uneffektive) Vor- und Nachbereitung aufgewendet werden musste. Folglich waren die Labore allesamt so eingerichtet, dass alle benötigten Instrumente sich darin befanden und keine weiteren Instrumente aus anderen Orten herbeigeschafft werden mussten. Denn nicht nur die Zeit der Aufbereitung war langwierig, auch die Arbeit am lebenden Organismus ließ sich nicht immer hinauszögern. So konnte an einem herausgetrennten Organ beispielsweise bloß in einem kalten Raum gearbeitet werden - mit einer Zentrifuge zu arbeiten, die plötzlich aus einem körperwarmen Raum hergebracht wurde, konnte auf das Organ zerstörerisch oder zumindest sehr irritierend wir- 


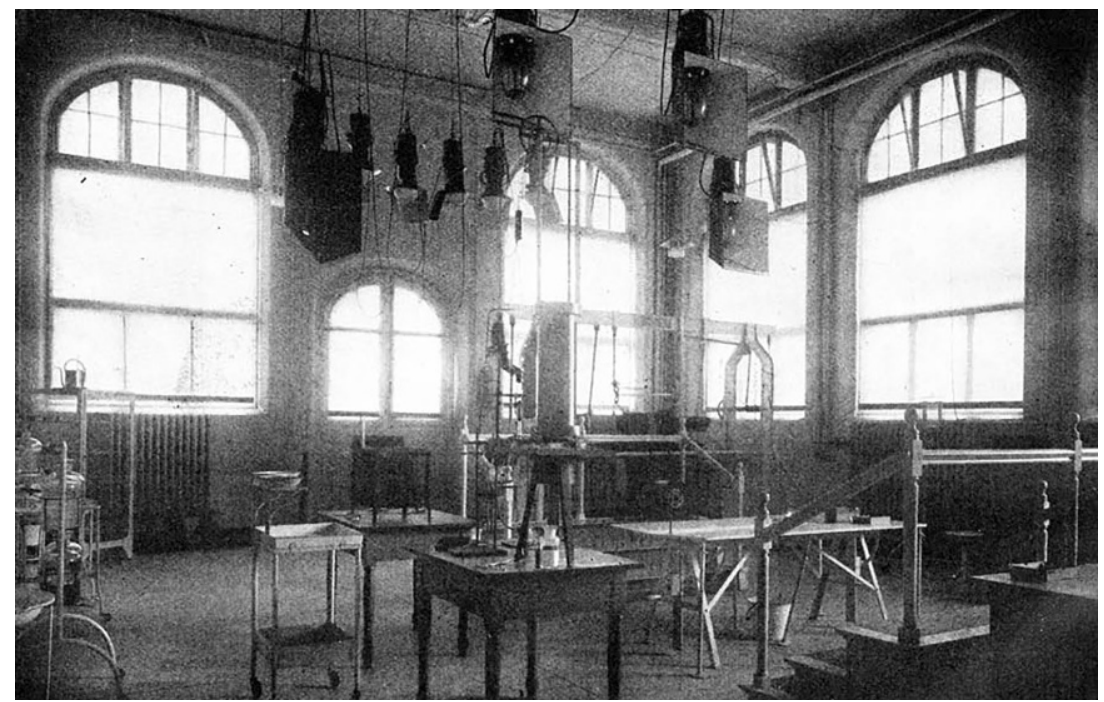

Abb. 2 Raum für Operationen und physiologische Experimente, de Haan: University of Groningen: Physiological Institute: 44

ken, sodass ein Abbruch des Experimentes stattfinden musste. Dabei ging es nicht bloß um die verschwendete Zeit und das verschwendete „Material", sondern natürlich in erster Linie um das lebendige Versuchsobjekt, das nicht einfach aus groben Fehlern geopfert werden sollte (ibid.: 38).

Ab 1925 umfasste Buytendijks Mitarbeiterkollegium etwa zwanzig Personen, darunter Mitarbeiter*innen für die Pflege, fünf Assistent"innen, darunter drei Freiwillige, die im Labor und bei der Betreuung der Studierenden aushalfen, gleichzeitig aber auch an ihren Forschungen arbeiteten; außerdem eine/n Mitarbeiter*in, die/der für die administrative Arbeit zuständig war, sowie ein Team von technischen Assistent*innen, zwei davon, die sich um die Instrumente und Apparate kümmerten und diese warteten, und zwei Personen, die bei den Experimenten mit den Apparaten halfen; daneben noch eine/n landwirtschaftliche/n Mitarbeiter"in, eine Reinigungskraft und zwei Lehrlinge. Das Budget für die Arbeit am Institut belief sich auf etwa 49.000 Niederländische Gulden. Man erkennt, dass Buytendijk seine Experimente nicht im Alleingang sondern in einem Team von Forschern durchführte. Neben seinem Stab an Mitarbeitern, wurde das Institut häufig auch von Gastwissenschaftlern besucht, wobei die Personen von ausgezeichneten Doktoranden anderer Kollegen (etwa Karl Duncker (1903-1940), Doktorand von Wolfgang Köhler (1887-1967) und Tamara Dembo (1902-1993)) bis hin zu Nobelpreisträgern (wie etwa August Krogh (1874-1943)) reichten. ${ }^{5}$ 
Im dargestellten institutionellen Kontext samt seinen experimentellen Möglichkeiten konnte Buytendijk epistemische Fragen gezielt und konstruktiv angehen. Dadurch verfestigte sich seine These, dass jede Reduktion, Vereinfachung oder Vereinseitigung wie beispielsweise die behavioristische Auffassung eine unzureichende Analyseweise des Lebendigen sei, da sich das Lebendige aus vielen variablen und unterschiedlichen Parametern konstituiert, nicht zuletzt im Austausch mit seiner natürlichen Umwelt, in die hinein es geboren ist. Sein eigenes Forschungsprogramm demonstrierte Buytendijk dem Kollegenkreis in seiner Antrittsvorlesung. Diese soll nachfolgend vorgestellt werden, bevor auf Buytendijks praktische Arbeit eingegangen wird.

\section{Buytendijks alternatives Forschungsprogramm}

In seinem Geleitwort zur Übersetzung von Buytendijks Groninger Antrittsvorlesung, schreibt der Botaniker und Philosoph Hans André (1891-1966) 1925:

Es ist gewiß kein Zufall, daß zum Leiter des dortigen Laboratoriums, des größten des Kontinents, ein Mann wie Buytendijk berufen wurde. Sein Vorgänger Hamburger war einer der Hauptvertreter der mechanistischen Schule in der Physiologie, während Buytendijk durch seine aufsehenerregenden Arbeiten, speziell auf dem Gebiet der Tierpsychologie, ein Führer der vitalistischen Methode zu werden verspricht. (Buytendijk 1925: 5)

Buytendijk bemühte sich mit dem Antritt der neuen Stelle um eine stärkere Integration der Tierpsychologie in die Physiologie, was bis dahin recht unüblich war. In den Augen der meisten Physiologen fiel die Tierpsyche nicht unter die Wissenschaft der organischen Funktionen als die die Physiologie galt. Obwohl, so Buytendijk, die meisten tierpsychologischen Untersuchungen doch nahelegten, dass das Tier und seine Umgebung eine gewissermaßen organische Ganzheit bildeten, so ließe „es sich schwer ableugnen, daß die auffallendsten Funktionen des tierischen Organismus jene Verrichtungen sind, welche Suchen, Ergreifen, Zerreißen der Nahrung, das Fliehen vor den Feinden, das Bauen und Aufsuchen von Verstecken, das Versorgen der Jungen usw. zum Zweck haben“ (ibid.: 23). Außerdem lieferten „die Methoden der Tierpsychologie die angemessene Technik für das Studium der Sinnesfunktionen und ihrer Lokalisation im Zentralnervensystem“ (ibid.), was für Buytendijk mindestens zwei Punkte ausmachte, 
die dafür sprächen, die Tierpsychologie in den größeren Fachbereich der Physiologie zu integrieren.

Frederik Buytendijk wurde 1925 zum Professor des physiologischen Institutes benannt, das seit seiner Erneuerung 1911 in den Händen des analytisch arbeitenden Physiologen Hamburger lag. Da Buytendijks Karriereweg bis dahin allerdings nicht ausschließlich von der analytisch-experimentellen Methode bestimmt war, sondern sich von der Humanmedizin hin zur Tierpsychologie und -physiologie entwickelte (van Buuren 2011; Prick 1974; Gruevska 2019b), legte er die Prioritäten, unter denen er künftig das renommierte Institut leiten wollte, weniger auf „eines der vielen sicherlich sehr interessanten Einzelprobleme [seines, Anm. d. V.] Faches“, sondern vielmehr auf die Erörterung der Frage, „ob die methodologischen Vorschriften der Physiologie eine Mehrung der Erkenntnis fördern" oder nicht (Buytendijk 1925: 10). Mit diesem in die experimentelle Praxis eingespeisten epistemologischen und wissenschaftsphilosophischen Programm beabsichtigte Buytendijks die „Lebenserscheinungen“, wie er sie nannte, $\mathrm{zu}$ erforschen - und zugleich eine neue Forschungsmethode und reflektierteres Denken in der Physiologie zu etablieren. Er hielt es für seine "Pflicht" sowie nicht zuletzt auch sein "gutes Recht", (ibid.) die Methoden der Physiologie nach philosophischen und epistemologischen Parametern zu hinterfragen. Dabei berief Buytendijk sich auf die Pionierleistung des Physiologen und Physikers Hermann von Helmholtz (1821-1894), der mit seinem eindrucksvollen Vergleich der Interdependenz von Theorie und Praxis über die Behandlung eines schwerkranken Patienten den Weg für dieses Recht geebnet habe (Helmholtz 1877). Die Verantwortung gegenüber Patienten und Angehörigen in der lebensweltlichen, alltäglichen Medizin, die angewandten therapeutischen Möglichkeiten $\mathrm{zu}$ rechtfertigen, mache es gleichsam notwendig darüber nachzudenken, „ob die Wissenschaft auch wohl alle Kenntnisse und Hilfsmittel vorbereitet habe, die sie hätten vorbereiten sollen, um zu wissen, daß erkenntnistheoretische Fragen über Methodik der Wissenschaft auch eine bedrängende Schwere und furchtbare praktische Tragweite erlangen können“ (ibid.: 6-7). In diesem Sinne stehe die Methode als Untersuchungsobjekt ebenso zur Disposition wie das Untersuchungsobjekt selbst. In der Interpretation Buytendijks erfuhr nun die „Induktion als der einzig richtigen Methode für physiologische Untersuchungen [bewußte Anerkennung]“, wobei die Deduktion einer „heilsamen Verwerfung“ anheimfiel (Buytendijk 1925: 11). Buytendijk diagnostizierte dieses Umdenken als ein Symptom der immer drastischeren Trennung von Empirie und Philosophie. Dabei erkannte er kritisch, dass sich „die Methode der Induktion zu jener der Analyse verengt" habe (ibid.). Zugleich stellte Buytendijk aber fest, dass es inzwischen Wunsch der Wissenschaftler sei, ihre Tätigkeitsbereiche zu öffnen (ibid.: 21), was er als nicht nur 
wünschenswert, sondern auch im hohen Grade vorteilhaft und gar notwendig erachte. Im Rückgriff auf historische Akteure wie Frans Cornelis Donders (1818-1889), Charles Darwin (1809-1882), Theodor Schwann (1810-1882), Jakob Moleschott (1822-1893) und Jacques Loeb (1859-1924) verwies Buytendijk darauf, dass es sich dabei zwar um ein altes, immer wiederkehrendes Problem handele, dies aber in ein ganz anderes Licht gerückt werde, „jetzt, wo von neuem die Frage sich aufdrängt, ob das Lebendige in seinen zusammengesetzten Formen und Funktionen vielleicht eine Vielheit von ursprünglichen Qualitäten besitzt, welche durch die Analyse zerstört wird“ (ibid.: 12). Den Vergleich der wissenschaftlichen Forschung mit einem Gemälde von Archibald V. Hill aufnehmend, wobei die einzelnen Farben das ganze Bild ausmachen, plädierte Buytendijk sowohl für eine Berücksichtigung der quantitativen Einzelheiten in der Forschung, als auch die qualitative Dimension, die die Einheit des Untersuchungsgegenstandes ausmacht. Dabei ging es Buytendijk nicht darum, vereinzelte kritische Punkte anzusprechen oder Methoden gar zu dekonstruieren, vielmehr sind es Hinweise für eine aufmerksamere Forschungsweise, die er einforderte, eine „innigere Vertrautheit mit der uns umgebenden Wirklichkeit“ (ibid.). So will er auch keine neuen Begriffe in die Lebenswissenschaften einführen, wie beispielsweise den zu dieser Zeit virulenten vitalistischen Begriff Hans Drieschs (1867-1941) der „Entelechie“; Buytendijk ging es vor allem um die „stärkere Betonung des ,Verstehens" neben dem ,Erklären““ (ibid.: 13). Denn, so Buytendijks Auffassung, „[d]ie Lebenserscheinungen können verstanden werden, und zwar sowohl als Handlung wie als Ausdruck“ (ibid.: 18). Dafür bedürfe es allerdings weder einer Auseinandersetzung mit den Methodenstreitigkeiten noch mit der rein philosophischen Debatte um die Leib-Seele-Problematik; vielmehr finde man ein solches Verstehen vor jeder psychologischen oder physiologischen Untersuchung, als gewissermaßen „vor- und außerwissenschaftliche[s] [Ausdrucksverstehen] “ (Buytendijk \& Plessner 1980 [1925]: 122). Explizit bindet Buytendijk diese Auffassung an die Ausführungen zur „Erklären-Verstehen-Distinktion“ des Geisteswissenschaftlers Wilhelm Diltheys (1833-1911) zurück („Die Natur erklären wir, das Seelenleben verstehen wir“, Dilthey 1924 [1894]: 144). Diese Dublette begreift das Leben als „psycho-physische Einheit“ (Dilthey 1924 [1894]; Scheler 2000 [1913-1916]: 157) und wird besonders in der philosophischen Anthropologie proklamiert (Plessner 1975 [1928], Scheler 2007 [1928]), an die Buytendijk anknüpft. In der Form einer „psycho-physischen Einheit" alles Lebendigen, wobei sich das Psychische als Ausdruck und Handeln über das Physische äußert, ist diese Auffassung korrelativ zum Verstehen und Erklären.

Die unterschiedlichen psycho-physischen Lebenserscheinungen präsentieren sich uns, Buytendijk zufolge, stets in einer Doppelgestalt: Erstens 
als eine Vielzahl von verworrenen Einzelheiten und zweitens als ein Ganzes. Die strikt analytische physiologische Methode kann eine generelle und drei konkrete Gefahren mit sich bringen, die vor Aufnahme einer experimentellen, häufig vivisektorischen Arbeit geklärt werden müssen. So ist „eine der ersten Pflichten des Physiologen, durch seine Versuchsmethoden aus dieser Einheit [der Lebenserscheinungen, Anm. d. V.] Elemente auszusondern" (Buytendijk 1925: 13). Die sich hieraus ergebende Schwierigkeit besteht darin, dass durch die Abtrennung, seien es die Trennungen von Organen oder Organismen, Nervenbahnen oder Hirnteilen, oder insbesondere die Trennung des Tierleibes von seiner Umwelt (worauf im weiteren Verlauf des vorliegenden Textes noch genauer eingegangen wird), "qualitative Einheiten zerstört werden können“ (ibid.). Konkret würden sich aus dieser übergeordneten Gefahr drei weitere ableiten, die zu einer falschen Erkenntnis über den Untersuchungsgegenstand führen könnten: Zum einen die Gefahr einer „unrichtigen Analyse“, falls beispielsweise die Trennung der Einheiten nicht entlang der funktionellen Zusammenhänge stattfände, sondern diese durchtrennt würden:

Die Kunst, die „natürliche“ Begrenzung der Teile zu finden, mag wohl in der Anatomie nicht so schwierig sein, jedoch die Begrenzung der Funktionen $\mathrm{zu}$ entdecken, das erheischt oft mühevolle Arbeit und ist durch die Art der Vorgänge selbst häufig ein Grund zu schier endlosen Irrungen. (Ibid.: 14)

Als zweite Gefahr führte Buytendijk die Missachtung der räumlichen und zeitlichen Kontinuitäten während fortgesetzter Untersuchungen an, infolgedessen die ursprünglichen Zusammenhänge aus den Augen verloren würden. Und drittens solle man versuchen, „in der Vielheit der elementaren Vorgänge die Einheit der Funktion“ (ibid.) erkennbar zu machen, indem man auf den richtigen Abstand zum Objekt achte. Das gelte auch bzw. in besonderem Maße für die Annahme, dass das Tier und seine Umwelt ebenso ein organisches Ganzes bildeten wie die Funktionen innerhalb des Tierleibes eine Ganzheit (ibid.: 21): „In der Morphologie ist das Verstehen der Form ein Suchen nach dem ,Bauplan', wobei das Tier als Einheit von ,Innenwelt und Umwelt' aufgefaßt wird.“ Ohne Uexküll explizit zu erwähnen, bezog sich Buytendijk auf dessen Theorie dahingehend, dass beide Ansätze in etwa nach den gleichen Mustern, wenn auch mit abweichender Terminologie funktionierten. Buytendijk verwendete die Begriffe "Situation“ (Buytendijk 1931) für das Geschehnis der Wechselwirkung von Tier und Umwelt sowie „Tierwerdung“ für den ständigen Vollzug dieser Interdependenz von Tier und Umwelt (Buytendijk 1958 [1929]). Um diese theoretischen Begrifflichkeiten sowie Buytendijks Umweltkonzeption ver- 
stehen und einordnen zu können, soll auf die praktischen Experimente eingegangen werden.

In seinem Text zur Methode der Beobachtung von Ratten in aufgabefreien Situationen (1931) kritisiert Buytendijk vor allem das ständige Bestreben der exakten Wissenschaftler alle Beobachtungen und Resultate auf eine schöne Zahl reduzieren zu müssen, überspitzt formuliert, ginge es sogar so weit, dass bestenfalls nur noch mit "ja“ oder "nein“ auf wissenschaftliche Fragen geantwortet werden solle. Selbst bei der Erforschung tierischen Verhaltens, das sich als besonders vielschichtig und mannigfaltig darstelle, bestünde der Anspruch, die Ergebnisse der Beobachtungen und Experimente in Tabellen festzuhalten und berechenbar zu machen. Da dies bei der Beobachtung von Tieren in der freien Wildbahn, aber auch im Labor, so gut wie unmöglich sei, würden die Versuchsanordnungen und Experimentierräume, so Buytendijks Kritik, immer reduktionistischer und eingeschränkter. Für die gewünschten eindeutigen Resultate verengten die speziell angefertigten technischen Laborräume einen ganzheitlichen Blick auf das Tier. Geblendet von ihrem „Exaktheitsideal“, sei die „Einfachkeit [sic!] von Versuchsbedingungen die Hauptsache“ (Buytendijk 1931: 574). ${ }^{6}$ Die gefährliche Konsequenz, die aus dieser Art der Forschung resultiere, sei, dass das wahre Wesen des Tiers als (psycho-physisches) Lebewesen übersehen und übergangen würde. Der falsche Anspruch der Exaktheit die Reduktion der Ergebnisse auf klare Zahlen und Tabellen zu bringen - würde der Tierpsychologie zum Verhängnis (Buytendijk 1927). Denn anstatt Erkenntnisse vom Verhalten der Tiere zu erlangen, würden diese mehr und mehr verdeckt (Buytendijk 1958 [1929]). Buytendijk stellt dagegen heraus, dass es gerade die Mannigfaltigkeit der Verhaltensweisen der Tiere sei, die ihr Verhalten und ihre Psyche kennzeichnen. Hierbei spielten sowohl ihre Instinkte und Reaktionen als auch Einsichten, Erfahrung und die untrennbare Einheit zu ihrer Umwelt eine wichtige Rolle (Buytendijk \& Fischel 1931).

Immer wieder führt Buytendijk - sowohl in seinen biophilosophischen wie in seinen fachspezifischen Aufsätzen - den russischen Physiologen Ivan Pawlow oder die US-Behavioristen um John B. Watson (1878-1958) und Karl S. Lashley (1890-1958) als Beispiel an und demonstriert an deren Versuchsanordnungen seine Kritikpunkte. Die Beanstandung des Experimentators Buytendijk bezieht sich somit auf die Theorie wie auch auf die technisch-reduktionistischen Experimentalumwelten, die das Tier „in einer unnatürlichen Lage, fest eingezwungen in einem Gestell [...] auf, einfache Reize reagieren [lassen] und zwar mit einer ,messbaren' Speichelabsonderung" (Buytendijk 1931: 574). Ein solches Verhalten kann und würde, so Buytendijk, in der natürlichen Umgebung des Hundes anders verlaufen, da die Versuchsanordnungen bereits an sich artifiziell sind. Damit evo- 
ziere man fahrlässig ein unnatürliches Verhalten des Hundes. So sei zum einen beispielsweise das „Gehen nach einem optischen Merkmal, oder Reagieren auf einen Ton für das Tier im allgemeinen unadäquat", und zum anderen die von der quantitativen Methode "geforderte ständige Wiederholung“ desselben Experimentes ein dauerndes „Eingreifen“ in den „Ablauf des inneren Tierlebens“, welches, so Buytendijks Kritik, „gewöhnlich bei solch einer Art des Experimentierens ausser Acht gelassen wird“ (ibid.: 575). Buytendijk kritisierte ebenfalls, dass die Annahmen und Vorurteile, die durch solche Versuche angestellt und weiter tradiert würden, sowohl die Tierpsychologie als auch primitivere Tiere in ein falsches Licht rückten. Man bekomme ein trügerisches Bild von den Versuchstieren, wenn man sie rein in ihrer Aufbewahrung, in der sie gezüchtet, gehalten und untersucht werden, denke, und nicht aus ihrer natürlichen Umgebung heraus. Denn in der natürlichen Umwelt sind Tiere sehr wohl zu „spontanen Leistungen fähig“ (ibid.: 567), ${ }^{7}$ wie Buytendijk aus langjähriger Erfahrung wußte (Buytendijk 1958 [1929]). ${ }^{8}$

\section{Konzeptualisierung der Tier-Umwelt-Einheit aus der experimentellen Forschung}

Unter dem Vorbehalt, dass Tiere nur als Einheiten von Organismus und Umwelt betrachtet werden können, stellt Buytendijk in seinen Texten häufig die Frage, inwiefern Experimente mit Tieren in Laboratorien, wo sie von ihrer Umwelt abgeschnitten sind, überhaupt Einsichten in das Leben der Tiere liefern können. Da es sich ja mit der Einheit des Tiers mit seiner Umwelt wie mit der Einheit des funktionellen Organismus verhalte, seien folglich auch alle anderen daraus gewonnenen Erkenntnisse verfälscht. Die Situationen, denen das Tier ausgesetzt ist, beispielsweise in T-Labyrinthen oder in Apparaturen (wie der berühmte pawlowsche Hund), ähneln in keiner Weise seinem natürlichen Habitat. Deshalb versuchte Buytendijk in seinen Experimenten ein alternatives Modell von Versuchsanordnungen auszuprobieren und zu etablieren: einen Versuchsraum, der dem natürlichen Lebensraum der Tiere etwas näherkam. Im Folgenden sollen zwei Experimente (mit Ratten und Hunden) vorgestellt werden, die Buytendijk unter anderem gemeinsam mit der Gestaltpsychologin Tamara Dembo initiiert hatte und aus denen er die Vorteile eines solchen geöffneten Versuchsraums ableitete. Zudem sollten diese einerseits seine Theorie der Tier-Umwelt-Einheit stützen sowie andererseits gerade seine Position gegen den US-amerikanischen und sowjetischen Behaviorismus verdeutlichen. 
1931 erschien in der physiologischen Fachzeitschrift Archives néerlandaises de physiologie de l'homme et des animaux unter dem Namen Buytendijks der bereits erwähnte Aufsatz „Eine Methode zur Beobachtung von Ratten in aufgabefreien Situationen“, mit dem Zusatz "(Nach Versuchen von Tamara Dembo)“ (Buytendijk 1931). Aus der Korrespondenz zwischen Buytendijk und Dembo in Buytendijks Nachlass erfahren wir, dass die Rattenversuche in diesem Text vor allem von Dembo, einer Doktorandin Kurt Lewins (1890-1947), während ihres Gastaufenthaltes in Groningen durchgeführt wurden (vgl. Lück 2011; Dekkers 1995). Da sie jedoch aufgrund ihres zeitgleich stattfindenden Examens sowie einiger Reisen und der Erkrankung ihres Vaters nicht dazu kam das Material auszuwerten, übernahm Buytendijk diese Aufgabe und machte sich schließlich die Experimente für seine Theorie zu Nutze, obwohl er eigentlich darauf bestand, dass die wichtigen Ergebnisse unter Dembos Namen veröffentlicht würden (van der Veer 2000). ${ }^{9}$ In den "Luna-Park"-Experimenten, wie Dembo sie in den Briefen bezeichnete, ${ }^{10}$ ging es darum, Ratten, ähnlich einer Untersuchung im Freiland, zu beobachten, ohne dass von der Experimentatorin Aufgaben vorgegeben waren. Dennoch erkannte Buytendijk die Vorteile des Beobachtungsexperimentes im geschlossenen (nicht eingeschränkten!) Raum, deutlich. Man könne schließlich

Ratten und andere Tiere im Freien schlecht beobachten. Dann liegt der Sinn des Experimentes aber auch darin, dass die Struktur der Situation in den Händen des Experimentators liegt, das Tier in einem vorher bestimmten Moment in die Situation hineinkommt und der ganze Verlauf sich vor dem Beobachter abwickelt. (Buytendijk 1931: 578)

Außerdem wäre ein Vergleich mehrerer individueller Tiere möglich, insofern diese in die immer gleiche Situation gebracht werden können, ohne an plötzliche umweltliche Veränderungen zu geraten. So wurden drei unterschiedliche Versuchsanordnungen aufgebaut, wobei der Raum in dem die Ratten hineingesetzt wurden mit seinen Maßen „von $2 \times 2 \mathrm{M}$ Grösse und $1 \mathrm{M}$ Höhe, der aus 4 gleichen Wänden und einer grösseren Bodenplatte aus Asbest zusammengestellt war" (ibid.), geräumig und mit vielen verschiedenen Dingen ausgestattet war (wie etwa zum Teil mit lebenden Fröschen oder Kaninchen), die für die Ratte interessant sein könnten (vgl. Abb. 3). Buytendijk zufolge bestand das "Geschick des Experimentators bei dieser Methode des Experimentierens [...] in der Erfindung immer neuer Situationen, die zum grossen Teil durch die Veränderungen der Inneneinrichtungen zustande gebracht wird“ (ibid.).

Die angestellten Beobachtungen waren erstaunlich: Obgleich die Versuchsratten 24 Stunden vor den Experimenten nicht gefüttert wurden, war 

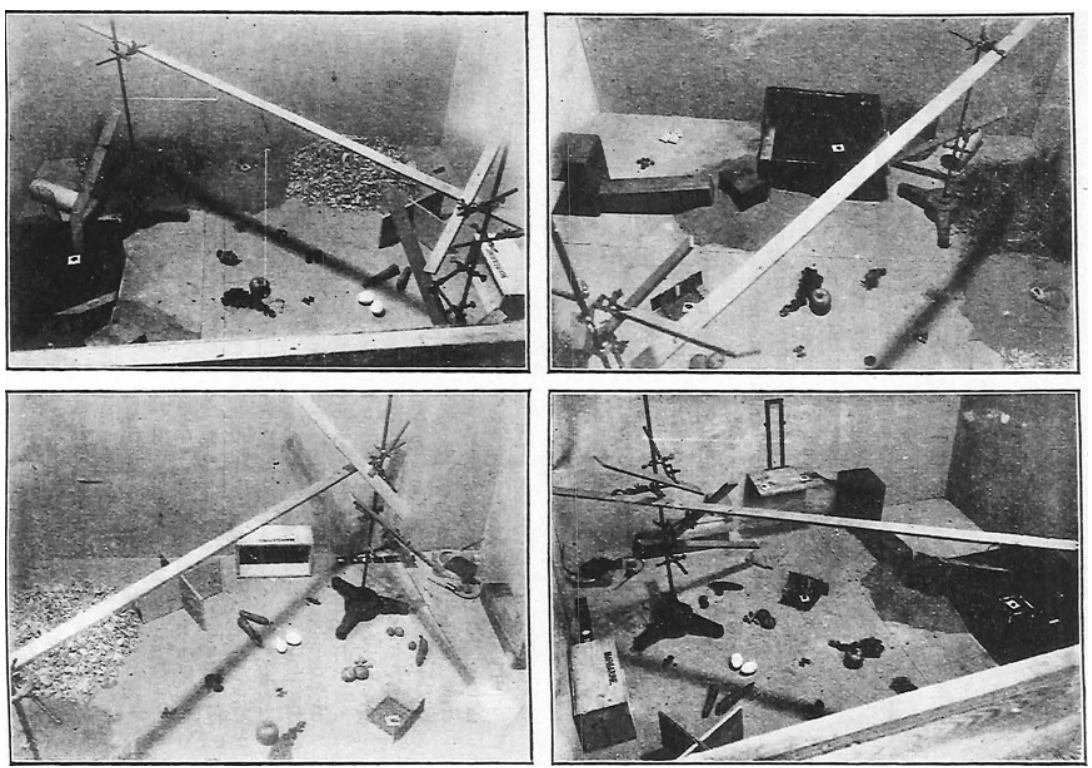

Abb. 3 Übersichtsbilder von Beobachtungskästen, Buytendijk (1931: 580)

ihre erste Intention nicht die Suche nach Nahrung, sondern die „neugierige“ Erkundung der Umgebung und der „vorläufigen Erfassung von Grenzen“:

Charakteristisch ist es dabei, dass nach erstmaligem Herumlaufen, das Verhalten sich sehr oft abrupt ändert: lief erst die Ratte nur wie im Vorübergehen schnüffelnd an den Wänden entlang, so geht sie jetzt in die Mitte des Käfigs zu den einzelnen Gegenständen hin. Der Raum ist von vornherein für die Ratte gegliedert, es gibt ein „längs den Wänden“, es gibt „ausgezeichnete Ecken“ (da wird in Ruhe gesessen). (Ibid.: 581)

Das Verhalten, das die Ratten mit Eingewöhnung in ihre Umgebung zeigen, war für Buytendijk besonders interessant, da sie in diesen freien Umgebungen, in denen sie nicht passiv einer Aufgabe unterstellt waren, zielgerichtetes Handeln ausführten und daran erkennbar wurde, „zu welchen Leistungen die Ratte eigentlich im Stande ist" (ibid.: 585). Teilweise verhielten sie sich einsichtig, staunend oder zweifelnd, beschreibt Buytendijk, wohl wissend, dass es sich hierbei um Anthropomorphismen handele (ibid.: 588). Dennoch seien dies wichtige Erkenntnisse, selbst wenn sie nur durch den Vergleich und mit den Begriffen der menschlichen Psyche operierten. Da sie das facettenreiche Verhalten von niederen Säugern - in diesem Fall: Ratten - zeigten, seien sie von großem Wert. Im Gegensatz zur „unnatürlichen Lage“ des pawlowschen Hundes, der sich „fest 
eingezwungen in einem Gestell“ befindet, sind die Ratten in Buytendijks und Dembos Experimenten mit einer zwar immer noch technischen, aber kontingenten, sich wandelnden und großzügigeren Umwelt konfrontiert, die ihr natürliches Verhalten zutage bringen soll. Pawlows Hund kann gar nicht anders reagieren, als von den Versuchsleitern beabsichtigt (ibid.: 574). In der Gegenüberstellung dieser beiden Versuchsanordnungen und ihren technischen „Umwelten“ wird deutlich, dass sich auch die epistemische Erwartungshaltung an das Experimentalobjekt ändert: Wo das Tier bei den Reflexversuchen „immer [...] etwas lernen [soll], als ob dies seine einzige psychologisch zu verstehende Leistung sei“, da kann bei Buytendijks und Dembos Versuchen „weit mehr beobachtet und beurteilt werden“ (ibid.: 596).

Wie aber wird eine solche Beobachtung operationalisiert, wenn sie nicht bloß in eine beschreibende Deskription des Experiments münden, sondern faktische Urteile über den Untersuchungsgegenstand durch das Verstehen der Experimentator*innen generieren soll? Unter welchen Prämissen kann diese Art der Experimentauswertung stattfinden, ohne dass Objektivität eingebüßt wird?

Buytendijk hatte eine spezifische Vorstellung davon, wer überhaupt solche Urteile vorzunehmen im Stande sei und in welchen Prozessen solche Urteile und Thesen entstünden. In einem Versuchsprotokoll zur Untersuchung des Stell- und Stemmbeinreflexes an hirnoperierten Ratten fasst Buytendijk seine Idee für ein alternatives Forschungsprogramm wie folgt zusammen:

In short for those, who frequently observed rats in many situations, their entire action is "imaginable". I believe that this simply means, that by frequently observing the actions of a certain kind of animals [sic!] in many different situations, we understand by feeling ourselves in the structure of the movemental patterns of the motion, how the action has been adapted to the internal and external position of the animal. As any other observation, we can transpose our observations of the conduct of animals to a new situation and therefor expect some definite method of proceeding, i.e. not an automatically accurate issue, but a certain relation of the speed and direction of the motion in connection of the environment. ${ }^{11}$

Aussagen über Tiere würden erst in der Beobachtung mit ihrer Interaktion zur Umwelt belastbar. Dafür sei es aber notwendig, einen erfahrenen Naturforscher mit dieser Aufgabe zu betrauen, jemand der sich lange Zeit und intensiv mit der Untersuchung und Beobachtung einer bestimmten Spezies beschäftigt habe. Erst diese Intensität und Erfahrung ermöglicht 
einen Aussagewert über Tiere. Versuchsleiter (vor allem Behavioristen), die nicht diese Hingabe zum Untersuchungsobjekt hegten, weil sie die Tiere als Gegenstände und nicht als Lebewesen betrachteten, könnten folglich durch eine reine quantitative Datenerhebung keine wirklichen Erkenntnisse vom Tier erlangen. Neben dieser wissenschaftstheoretischen Kritik an der analytisch arbeitenden Physiologie erkannte Buytendijk noch in anderer Hinsicht, in welchem hohen Maße das Tier mit seiner Umwelt verbunden ist.

Das nachfolgend dargestellte Experiment ist hierfür besonders interessant, weil Buytendijk in diesem aus zwei Teilen bestehenden Versuch, den er an Hunden durchführte, dieselbe Vorrichtung nutzte, wie Pawlow in seinem berühmten Experiment. Damit griff Buytendijk erneut die statische Experimentalumwelt an und stellte dieser seine offenere Konzeption entgegen. Obgleich Buytendijk in seinem Experiment nicht explizit kritisch auf die Versuche Pawlows eingeht, wie in seinen späteren Texten ab 1929, verdeutlicht dieser Versuch dennoch, welche Beobachtungen Buytendijk zur harschen Kritik an Pawlow veranlassten.

Im Jahre 1924, noch bevor Buytendijk als Direktor in Groningen tätig war, führte er in Amsterdam, wo er zuvor die Leitung des dortigen physiologischen Institutes innehatte (1919-1925), ein Experiment zur „Formwahrnehmung beim Hunde" (Buytendijk 1924) durch. Damit initiierte er eine Versuchsreihe, die an ähnliche Hundeexperimente anknüpfte, so zum Beispiel an Sir John Lubbocks (1834-1913) Versuch seinem Pudel beizubringen, die Form einzelner Wörter zu erkennen (Lubbock 1884a; 1884b). Buytendijks Anliegen war es nun, herauszufinden, ob Hunde auch abstrakte, sprich: mathematische Figuren erkennen und voneinander unterscheiden können. Anhand dieser Erkenntnis ließen sich sodann, so seine Hoffnung, drei weitere Fragen klären: Erstens, inwiefern ein optischer Sinn beim Hund ausgeprägt ist, galt dieser doch zumeist als „Geruchstier“. Zweitens, ob der Hund eventuell nur bewegliche Objekte erfasst und drittens, ob Zusammenhänge zwischen der Wahrnehmung und dem Erkennen von Formen und der Einheit von Tier und Umwelt bestehen. Die erste der beiden Untersuchungen wurde mit einem Hund angestellt, der mit einem multiplen Wahlapparat konfrontiert wurde (s. Abb. 4); der zweite Versuch mit einem Hund, der an eine der pawlowschen ähnliche Apparatur fixiert wurde (s. Abb. 5). So war es dem ersten Hund möglich, sich im Raume $\mathrm{zu}$ bewegen, dem zweiten hingegen nicht. Buytendijk selbst beschrieb die erste Versuchsanordnung wie folgt:

Der von uns benutzte Apparat ist in Abb. 1 [hier: Abb. 4, Anm. d. Verf.] abgebildet. Er besteht aus einem Kasten (a) mit Gitterabschluß dem fächerförmigen Raum gegenüber. Der Bogen enthält 12 viereckige Öff- 


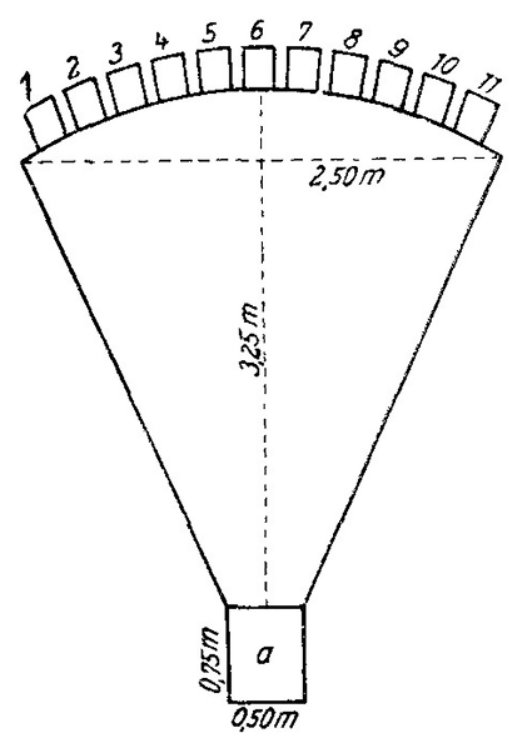

Abb. 4 Multipler Wahlapparat, Buytendijk (1924: 7)

nungen, die mit kleinen Klapptüren aus Zink verschlossen sind. Der Hund kann eine von diesen Türen wählen, mit der Nase aufstoßen, und findet hinter der Tür in einem Kästchen Nahrung. Die anderen Türen können unsichtbar verriegelt werden. [...] In den Vorversuchen habe ich [Buytendijk, Anm. d. V.] das Tier an den Apparat gewöhnt, indem die Tür, an welcher die Figur angebracht ist, in dem Moment geöffnet wird, in welchem der Hund in die Nähe kommt. [...] In meinen (Buytendijks, Anm. d. V.) Versuchen habe ich einen Hund auf ein Dreieck dressiert. Die Figur war, wie auch alle anderen der ersten Versuchsreihen, mit Tusche aus $3 \mathrm{~mm}$ dicken Strichen auf weißem Karton gezeichnet. (Buytendijk 1924: 8)

Bei der ersten Versuchseihe wurde das Tier zunächst an den Wahlapparat gewöhnt, indem nur wenige Klappen sichtbar gemacht wurden. Bereits nach fünfzehn Versuchen hatte der Hund die Tür mit dem Keks dahinter problemlos gefunden. Später wurden auch die Figuren getauscht, sodass das Dreieck immer an einer anderen Klappe befestigt war. Auch hierbei lernte der Hund rasch, das Dreieck zu finden, selbst wenn die Dreiecke kleiner gedruckt waren als zuvor. So ging der Hund in einem Abstand von etwa $30 \mathrm{~cm}$ an den Klappen vorbei, bis er das Dreieck fand. „Besonders auffallend war Versuch 65", hebt Buytendijk hervor: 


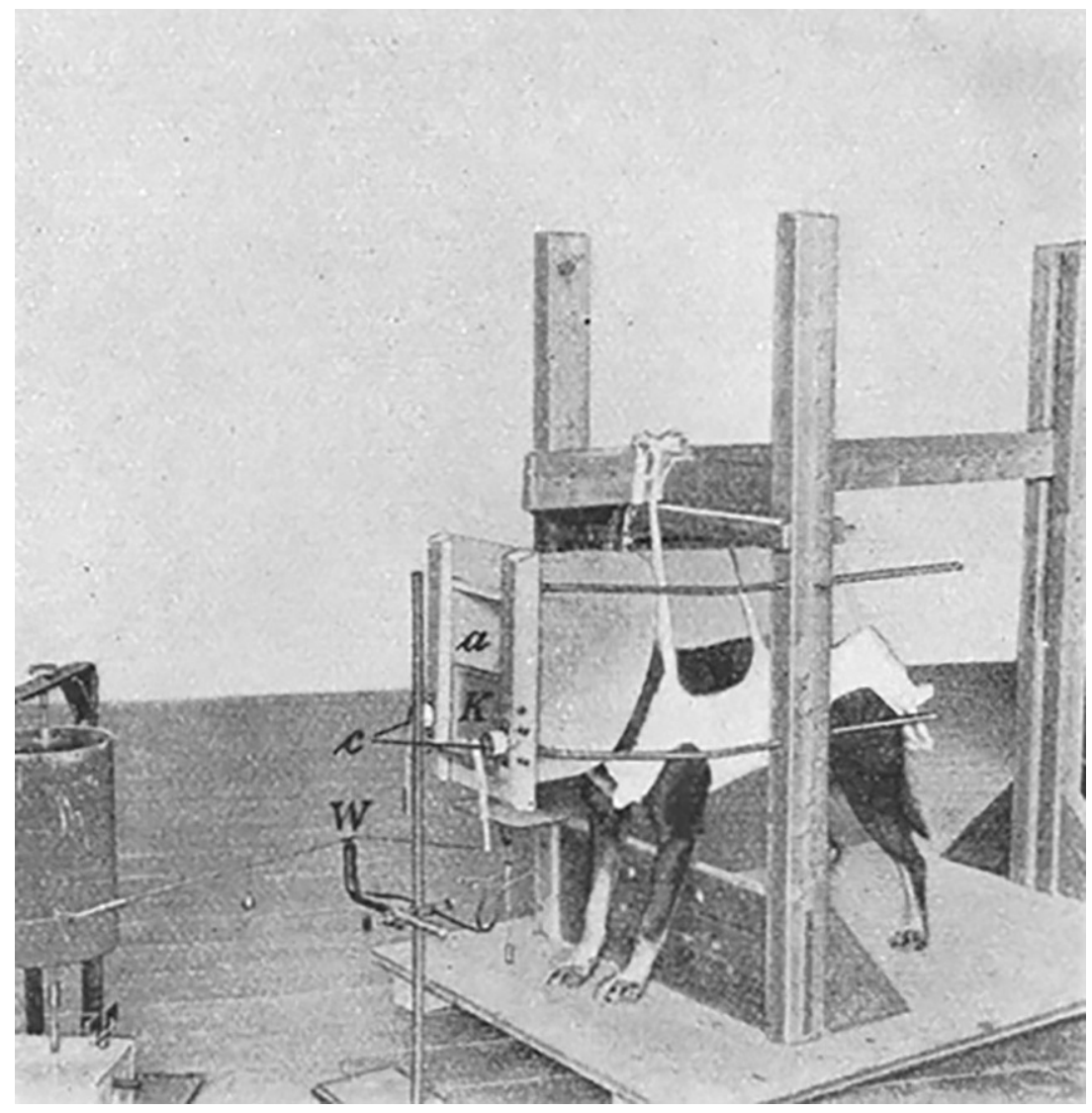

Abb. 5 Versuchsanordnung der zweiten Versuchsreihe, Buytendijk (1924: 12)

Unter den bezeichneten Türen war das Dreieck ausgelassen. Das Tier ging mehrmals die Reihe der Klappen entlang, lief dann im Apparat herum und kehrte schließlich spontan zum Kasten a wieder zurück, wie es auch in den anderen Versuchen nach enthaltener [sic!] Nahrung zu tun gewöhnt war. Die nächste Probe war wieder eine normale Anordnung, wobei der Hund die Auswahl richtig traf. (Ibid.)

Damit stellte Buytendijk bereits vorläufig fest, dass ein „Hund unter den gegebenen Umständen sehr wohl imstande ist, eine mathematisch einfache Figur unter anderen zu ,erkennen“" (ibid.: 11). Diese These bestätigte sich für Buytendijk komplett, nachdem der Hund außerdem lernte das Dreieck zu erkennen, selbst wenn es beispielsweise gedreht wurde und mit der Spitze nun nach unten oder zur Seite zeigte. Dies lernte der Hund aber erst, nachdem er mit den kleineren Dreiecken konfrontiert worden war. 
Der zweite Versuch wurde mit einem Hund durchgeführt, der in einer Apparatur fixiert wurde (s. Abb. 5):

Die am Anfang auftretende Aufregung war nach einigen Tagen schon dermaßen verschwunden, daß der Hund sich ohne Widerstreben befestigen ließ und keine Befreiungsversuche machte. Gegenüber dem Fenster a befand sich auf $1 \mathrm{~m}$ Abstand ein weißes angespanntes Tuch, auf welchem wir die Figuren projizierten. Wenn die Reizfigur exponiert wurde, konnte der Hund die Klappe (k) unter dem Fenster (a) mit der Nase aufstoßen und das Futter aus dem Behälter nehmen. [...] Unsere [Buytendijks, Anm. d. V.] Untersuchung bezweckte die Einstellung des Hundes auf ein vorgezeigtes, sich nicht bewegendes Dreieck. (Ibid. 11f.)

Die Ergebnisse dieses Versuchs waren einerseits ernüchternd, andererseits hochinteressant, weil sie Buytendijks kritische Haltung gegenüber Pawlow stärkten und die Gefahren der eingeschränkten Versuchsanordnungen zeigten: Der Hund hatte nach tausend Versuchen in fünf Monaten keine Lernerfolge erzielt und die Form des Dreiecks nicht erkannt. Diese eindrucksvolle Beobachtung ließ Buytendijk die These aufstellen, „daß die Beziehung von Innenwelt und Umwelt der Tiere nicht so gedacht werden kann, daß eine Einpassung des Tieres in seine Umgebung nach der Art der Einpassung eines Schlüssels ins Schloß vorliegt" (ibid.: 13). Außerdem zeige die Formwahrnehmung, dass der Hund tatsächlich dazu in der Lage sei, abstrakte Formen wiederzuerkennen. Eine Fähigkeit, die Buytendijk als „sensorische Begriffsbildung“ bezeichnete und analog zum menschlichen Denken verstand - nur in anderen Dimensionen und Inhalten. Um seine These einzubetten, gab Buytendijk seine Beobachtungen sinngemäß mit den Worten Uexkülls wider: „Die Bewegung auf den optischen Eindruck hin bildet ein integrierendes Moment in der Melodie der Umwelt, wodurch auch die Form der Gegenstände in der Innenwelt zum Erklingen gebracht werden“ (ibid.: 14). So ist die „Einpassung“ von der er spricht, zwar auch von Uexküll entlehnt, diese geschieht bei Buytendijk aber erst über das Medium der Bewegung, weshalb er nicht von einer Einpassung im Sinne des „Schlüssel-Schloss-Prinzips“ ausgeht. Uexkülls „Einpassung“ des Tiers in seine Umwelt wird in der Forschung allerdings sehr wohl als adäquat zum Schlüssel-Schloss-Prinzip verstanden. Bei Buytendijk kommt in der Interaktion von Tier und Umwelt eine mediale Komponente hinzu: Die Dynamik des Lebewesens in der Interaktion mit seiner Umwelt ermöglicht überhaupt erst diese wahrzunehmen und mit ihr in Kontakt zu treten. Erst in dieser Interaktion, in der aktiven, dynamischen Wechselwirkung mit der Umwelt wird das Tier zu einer Ganzheit - zum Tier. 


\section{"Tierwerdung" - ein Kulminations- und wissenschaftsphilosophischer Aussichtspunkt}

"Tierwerdung“ - so bezeichnet Buytendijk seine spezifische Auffassung von der Dynamik der Wechselwirkung zwischen tierischem Leib und Umwelt. Die Umwelt ist dem Tier eigen, sie ist ihm ein „Organ“ (Buytendijk 1958 [1929]: 56), das ihm den Raum gibt, sich zu verhalten und lebendig zu werden. Die Umwelt ist ihm „nicht gegenständlich gegeben“, es hat „keine Vorstellung davon" (ibid.: 29), sondern sie ist ein ausgelagert-integrierter Bestandteil seines Organismus. Neben der unabdingbaren Verbindung von Wahrnehmung und Reaktion, die die Handlung des Tiers ausmachen, ist es gerade der "Affekt" als intrinsische Motivation, der die Einheit von Umwelt und Tier entstehen lässt. Die tierischen Affekte sind plural, so könne man darunter alle Instinkte sowie beispielsweise aber auch neugierige Handlungen fassen. In Buytendijks hier theoretisch wie konkret dargestellter Theorie erscheint der „den Tieren innewohnende Drang nach Selbst- und Arterhaltung [...] in den verschiedenen Affekten und ist der Grund für die Bildung der sensorischen und motorischen Umwelt, der ,Merk- und Wirkungswelt" (ibid.: 57). Damit sei die Relation zwischen Tier und Umwelt eine "notwendige und zweckmäßige“ (ibid.).

Es konnte in der Untersuchung gezeigt werden, dass es Buytendijk in seiner Forschung vor allem um die Frage ging, wie man das Problem des psycho-physischen Zusammenhangs, der sich in den Lebenserscheinungen bei den Tieren wie bei den Menschen zeige, methodisch darstellen könne. Dieses Interesse folgte dabei der Auffassung, dass die Physiologie analytisch schon die einzelnen Funktionen umfassend erforschen könne, und auch die Anatomie beträchtliche Erkenntnisse in der Unterscheidung von menschlichen und tierischen Körperbauten erzielt habe, diese jedoch nicht ausreichten, um die eigentliche Pluralität und Komplexität der Lebenserscheinungen in ihrer Wechselwirkung mit der Umwelt zu sensibilisieren. Diese zeige sich laut Buytendijk erst durch die Ganzheitsauffassungen der Lebenserscheinungen, also durch eine „harmonische Einsicht in diese Einheit stofflicher und geistiger Vorgänge“ (ibid.: 23), die aber von den analytischen Erfahrungswissenschaften größtenteils unbeachtet blieben. Buytendijk erkannte, wie in diesem Beitrag herausgestellt wurde, dass quantitative Forschungsmethoden bei entsprechender Fragestellung zwar Einblicke und Erkenntnisse bei der Untersuchung von Naturerscheinungen liefern können, jedoch eine ganzheitliche Auffassung, unter denen die Lebenserscheinungen stehen, damit nicht zu erfassen sei. Zur reflektierten und aufmerksamen Untersuchung dieser lebendigen Einheiten gehören neben den theoretischen (Uexküll, Phänomenologie, Hermeneutik) auch insbesondere die experimentellen Bedingungen, die Buytendijk für seine 
Versuche entwarf. Beide Voraussetzungen erlaubten es Buytendijk epistemologisch eine philosophisch-reflexive Fragestellung zu generieren, um der Natur der Tiere gerechter zu werden und diese in seinen Experimenten zu berücksichtigen. So erhielt er all seine Erkenntnisse zum Wesen der Lebenserscheinungen, nicht etwa durch vivisektorische Eingriffe, sondern mittels einer durch „experimentelle ,Hinweise $[. .$.$] phänomenologisch ge-$ wonnene[n] Einsicht" (ibid.: 38).

\section{Danksagung}

Ich bedanke mich recht herzlich bei den Gutachter*innen für die wertvollen Anmerkungen und Anregungen zu diesem Aufsatz.

\section{Anmerkungen}

11866 entwickelte Ernst Haeckel die „Ökologie“, obwohl dieser Begriff „jahrelang unbekannt [blieb]“ (Müller 2001: 99).

2 Der Unterschied zwischen Umgebung und Umwelt gestaltet sich bei Uexküll so, dass die Umgebung die objektive Wirklichkeit der Menschen darstellt, während sich das Tier seine eigene Umwelt konstruiert. Giorgio Agamben fasst die Unterscheidung folgendermaßen auf: „Uexküll unterscheidet mit Bedacht zwischen der Umgebung, dem objektiven Raum, in welchem wir ein Lebewesen in Bewegung sehen, und der Umwelt, die sich aus einer mehr oder weniger umfangreichen Reihe von Elementen zusammensetzt, die er Bedeutungsträger und Merkmalträger nennt und die allein für das Tier relevant sind. Die Umgebung ist in Wirklichkeit unsere eigene Umwelt, die von Uexküll mit keinerlei Privilegien versehen wird und die somit je nach Perspektive veränderbar ist" (Agamben 2003: 50).

3 BUYT-344. Archief F.J.J. Buytendijk. Katholiek Documentatie Centrum Nijmegen. Archiv-Dokumente werden, gemäß der Inventarnummer, im Folgenden als BUYT-XY wiedergegeben.

4 So ist nicht unbedingt davon auszugehen, dass beispielsweise Konrad Lorenz' Methode der Freilandbeobachtung eine Weiterführung im Sinne Buytendijks war, weil die natürlichen Gegebenheiten, die auf das Tier einwirkten oder denen das Tier ausgesetzt war, nicht vom Experimentator kontrollierbar und berechenbar waren. Für Buytendijk war die Kontrolle des Versuchsleiters über das Experiment eines der wichtigsten Parameter einer wissenschaftlichen Auseinandersetzung,

5 Vgl. BUYT-236.

6 Besonders im Blick hat Buytendijk die Assoziationspsychologie sowie die analytische Tierpsychologie.

7 „Alles, was die Ratte zu ihren Rattenangelegenheiten bewegt, das muss für die Psychologie von Wichtigkeit sein. Nur so kann die Grundlage einer wirklich vergleichenden Verhaltensforschung gelegt werden" (Buytendijk 1931: 576 f.). Versuchen solcher Art sei es demzufolge zu verdanken, dass man das Leben der niederen Säugetiere „als weniger mannigfaltig ansah, wie etwa das des Affen“ (ibid.: 575). 
8 So behandeln die Behavioristen und Assoziationspsychologen bzw. die Reizreflextheoretiker die Tiere nicht als Lebewesen, sondern lediglich als Material: Die Tiere werden nicht individuell beobachtet, und so hat „der Experimentator [...] z.B. keine Ahnung von dem Verhalten der Ratten ausserhalb [der] wenigen von Ihm ausgewählten Reaktionssituationen" (Buytendijk 1931: 575). Auch Buytendijk hat den Anspruch für die Forschung „exakt" zu sein, allerdings nicht um den Preis, damit das für ihn wahre Wesen des Lebendigen zu vertuschen. So postuliert er: „Der gewissenhafte Forscher soll seine Methoden der Grundstruktur seines Objektes anpassen" (ibid.: 567). Buytendijk erwartet von den Experimentatoren Flexibilität und ein Feingefühl für die Tiere, um den Tieren entsprechende Methoden zu entwerfen und Untersuchungen anzustellen, abzuwägen und zu reflektieren.

9 BUYT-157.

10 BUYT-157

11 BUYT-1355: $14 \mathrm{f}$.

\section{Literatur}

Agamben, Giorgio 2003. Das Offene. Der Mensch und das Tier. Frankfurt a. M.: Suhrkamp. Ballauff, Theodor, Eckart Scheerer und Ahlrich Meyer 1984. Organismus. In: Joachim Ritter und Karlfried Gründer (Hg.). Historisches Wörterbuch der Philosophie. Bd. 6. Basel: Schwabe: $1330-1358$.

Becker, Ralf 2015. Der Sinn des Lebens. Helmuth Plessner und F.J.J. Buytendijk lesen im Buch der Natur. In: Kristian Köchy und Francesca Michelini (Hg.). Zwischen den Kulturen. Plessners „Stufen des Organischen“ im zeithistorischen Kontext. Freiburg/München: Karl Alber: 65-89.

Block, Katharina 2016. Von der Umwelt zur Welt. Der Weltbegriff in der Umweltsoziologie. Bielefeld: transcript.

Bühler, Benjamin 2004. Tier, Experiment und Philosophische Anthropologie. In: Ulrich Bröckling, Benjamin Bühler, Marcus Hahn, Matthias Schöning und Manfred Weinberg (Hg.). Disziplinen des Lebens. Zwischen Anthropologie, Literatur und Politik. Tübingen: Narr: 47-60

Burgat, Florence und Christian Sommer (Hg.) 2016. Le phénomène du vivant. Buytendijk et l'anthropologie philosophique. Genève: Métis Presses.

Buuren, Jasper van 2011. Buytendijk und die Philosophische Anthropologie. Internationales Jahrbuch für philosophische Anthropologie (2): 285-300.

Buuren, Jasper van 2018. Body and Reality: An Examination of the Relationships between the Body Proper, Physical Reality, and the Phenomenal World Starting from Plessner and Merleau-Ponty. Bielefeld: transcript.

Buytendijk, Frederik J. J. 1924. Über die Formwahrnehmung beim Hund. Pflügers Archiv (205): 4-14.

Buytendijk, Frederik J. J. 1925. Über das Verstehen der Lebenserscheinungen. Habelschwerdt: Frankes Buchhandlung.

Buytendijk, Frederik J. J. 1927. Experimentelle Tätigkeit und Biophilosophie. In: Hermann Schneider und Werner Schingnitz (Hg.). Festschrift. Hans Driesch zum 60. Geburtstag. 1. Teil: Wissen und Leben. Leipzig: Reinicke: 77-87.

Buytendijk, Frederik J. J. 1931. Eine Methode zur Beobachtung von Ratten in aufgabefreien Situationen (Nach Versuchen Tamara Dembos). Archives Néerlandaise de physiologie de l'homme et des animaux (16): 574-582.

Buytendijk, Frederik J. J. 1958 [1929]. Zur Untersuchung des Wesensunterschieds von Mensch und Tier. In: Ders. (Hg.). Das Menschliche. Wege zu seinem Verständnis. Stuttgart: Koehler: 21-59.

Buytendijk, Frederik J. J. und Werner Fischel 1931. Versuch einer neuen Analyse der tierischen Einsicht. Archives Néerlandaises de Physiologie de l'homme et des animaux (16): $449-476$. 
Buytendijk Frederik J. J. und Helmuth Plessner 1935. Die physiologische Erklärung des Verhaltens. Eine Kritik an der Theorie Pawlows. Acta Biotheoretica (1/3): 151-171.

Buytendijk, Frederik J. J. und Helmuth Plessner 1980 [1925]. Die Deutung des mimischen Ausdrucks. Ein Beitrag zur Lehre vom Bewußtsein des anderen Ichs. In: Günther Dux, Odo Marquard und Elisabeth Ströker (Hg.). Helmuth Plessner. Gesammelte Schriften. Bd. VII: Ausdruck und menschliche Natur. Frankfurt a. M.: Suhrkamp: 67-129.

De Haan, Johann Abraham Bierens 1925. University of Groningen: Physiological Institute. Method and problems of medical education (3): 35-45.

Dekkers, Wim J.M. 1995. F.J.J. Buytendijk's concept of an anthropological physiology. Theoretical Medicine (16): 15-39.

Dilthey, Wilhelm 1924 [1894]. Ideen über eine beschreibende und zergliedernde Psychologie. In: Georg Misch (Hg.). Wilhelm Dilthey. Gesammelte Schriften. Bd. V. Leipzig/Berlin: Teubner: 139-240.

Fischer, Joachim 2009. Philosophische Anthropologie. Eine Denkrichtung des 20. Jahrhunderts. Freiburg i. Br.: Karl Alber.

Gruevska, Julia 2018. „Natürliches Verstehen.“ Phänomenologische Deutung und Erfahrung als Methode im Denken Helmuth Plessners und Frederik Buytendijks. In: Hans-Ulrich Lessing und Kevin Liggieri (Hg.). „Das Wunder des Verstehens." Ein interdisziplinärer Blick auf ein , außer-ordentliches' Phänomen. Freiburg i. Br.: Karl Alber: 104-125.

Gruevska, Julia 2019a. Das naturimitierende Labor. Philosophisch-anthropologische Implikationen in der Physiologie Frederik Buytendijks. In: Julia Gruevska (Hg.). Körper und Räume. Wiesbaden: Springer: 133-151.

Gruevska, Julia 2019b. Von der Tierphysiologie zur Psychologie des Menschen. Ein Einblick in Werk und Wirken Frederik Buytendijks. Internationales Jahrbuch für philosophische Anthropologie (8): 87-107.

Hartung, Gerald 2008. Philosophische Anthropologie. Stuttgart: Reclam.

Helmholtz, Hermann von 1877. Das Denken in der Medicin. Rede, gehalten zur Feier des Stiftungstages der militairärztlichen Bildungs-Anstalten am 2. August 1877. Berlin: Verlag von August Hirschwald.

Köchy, Kristian 2015. Helmuth Plessners Biophilosophie als Erweiterung des Uexküll-Programms. In: Kristian Köchy und Francesca Michelini (Hg.). Zwischen den Kulturen. Plessners „Stufen des Organischen" im zeithistorischen Kontext. Freiburg/München: Karl Alber: 25-64.

Lenoir, Timothy 1982. The Strategy of Life: Teleology and Mechanics in Nineteenth Century German Biology. Chicago: University of Chicago Press.

Lubbock, John 1884a. Teaching Animals to Converse. Nature (29): 216.

Lubbock, John 1884b. Teaching Animals to Converse. Nature (29): 547-548.

Lück, Helmuth E. 2011. Tamara Dembo: Auf der Suche nach Konzepten für ein besseres Leben. In: Helmut E. Lück und Sibylle Volkmann-Raue (Hg.). Bedeutende Psychologinnen des 20. Jahrhunderts. Berlin: Springer: 179-191.

Marquard, Odo 1971. Anthropologie. In: Joachim Ritter, Karlfried Gründer und Gottfried Gabriel (Hg.). Historisches Wörterbuch der Philosophie. Bd.1. Basel/Stuttgart: Schwabe: 362-374.

Mildenberger, Florian und Bernd Herrmann 2014. Nachwort. In: Dies. (Hg.). Uexküll: Umwelt und Innenwelt der Tiere, Berlin: Springer: 260-330.

Müller, Gerhard H. 2001. Umwelt. In: Joachim Ritter, Karlfried Gründer und Gottfried Gabriel (Hg.). Historisches Wörterbuch der Philosophie. Bd. 11. Basel: Schwabe: 99-105.

Müller, Ernst und Falko Schmieder 2008. Einleitung. In: Ernst Müller und Falko Schmieder (Hg.). Begriffsgeschichte der Naturwissenschaften: Zur historischen und kulturellen Dimension naturwissenschaftlicher Konzepte. Berlin: de Gruyter: XI-XXIII.

Nietzsche, Friedrich 1980 [1886]. Jenseits von Gut und Böse. Vorspiel einer Philosophie der Zukunft. In: Giorgio Colli und Mazzino Montinari (Hg.). Friedrich Nietzsche. Sämtliche Werke. Kritische Studienausgabe. Bd. 5. Berlin/New York: de Gruyter: 9-243.

Plessner, Helmuth 1975 [1928]. Die Stufen des Organischen und der Mensch. Einleitung in die philosophische Anthropologie. Berlin/New York: de Gruyter.

Plessner, Helmuth 1985. Selbstdarstellung. In: Günter Dux, Odo Marquard und Elisabeth Ströker (Hg.). Schriften zur Soziologie und Sozialphilosophie. Gesammelte Schriften. Bd. 10. Frankfurt a. M.: Suhrkamp: 302-341. 
Plessner, Helmuth 2019 [1961]. Philosophische Anthropologie. Göttinger Vorlesung vom Sommersemester 1961. Julia Gruevska, Hans-Ulrich Lessing und Kevin Liggieri (Hg.). Frankfurt a. M.: Suhrkamp.

Potthast, Thomas 2010. Umwelt. In: Philipp Sarasin und Marianne Sommer (Hg.). Evolution. Ein interdisziplinäres Handbuch. Stuttgart/Weimar: Metzler: 50-52.

Prick, J.J.G. 1974. Levensbericht F.J.J. Buytendijk. Jaarboeck. Amsterdam: 207-229.

Rheinberger, Hans-Jörg 2007. Historische Epistemologie zur Einführung. Hamburg: Junius.

Rheinberger, Hans-Jörg 2008. Begriffsgeschichte epistemischer Objekte. In: Ernst Müller und Falko Schmieder (Hg.). Begriffsgeschichte der Naturwissenschaften: Zur historischen und kulturellen Dimension naturwissenschaftlicher Konzepte. Berlin: de Gruyter: $1-12$.

Scheler, Max 2000 [1913-1916]. Der Formalismus in der Ethik und die materiale Wertethik. In: Maria Scheler und Manfred Frings (Hg.). Max Scheler. Gesammelte Werke. Bd. 2. Bonn: Bouvier.

Scheler, Max 2007 [1928]. Die Stellung des Menschen im Kosmos. Bonn: Bouvier.

Schmieg, Gregor 2017. Die Systematik der Umwelt: Leben, Reiz und Reaktion bei Uexküll und Plessner. In: Thomas Ebke und Caterina Zanfi (Hg.). Das Leben im Menschen oder der Mensch im Leben? Deutsch-Französische Genealogien zwischen Anthropologie und Anti-Humanismus. Potsdam: Universitätsverlag Potsdam: 347-360.

Spiegelberg, Herbert 1972. Phenomenology in Psychology and Psychiatry: A Historical Introduction. Evanston: Northwestern University Press.

Thines, Georges und René Zayan 1975. F. J. J. Buytendijk's contribution to animal behaviour: Animal Psychology or Ethology? Acta Biotheoretica (26/3-4): 86-99.

Toepfer, Georg. 2011. Umwelt. In: Georg Toepfer (Hg.). Historisches Wörterbuch der Biologie. Bd. III. Stuttgart/Weimar: Metzler: 566-607.

Toepfer, Georg 2017. Biologie und Anthropologie der Wahrnehmung. Kopplung und Entkopplung von Organismus und Umwelt. In: Gerald Hartung und Matthias Herrgen (Hg.). Interdisziplinäre Anthropologie (4). Wiesbaden: Springer: 3-49.

Uexküll, Jakob von 1909. Umwelt und Innenwelt der Tiere. Berlin: Springer.

Uexküll, Jakob von 1921. Umwelt und Innenwelt der Tiere. 2. verm. u. verb. Aufl. Berlin: Springer.

Van der Veer, Rene 2000. Tamara Dembo's European years: Working with Lewin and Buytendijk. Journal of the History of Behavioral Science (2): 109-126.

Wunsch, Matthias 2016. Was macht menschliches Denken einzigartig? Zum Forschungsprogramm Michael Tomasellos. Interdisziplinäre Anthropologie (3): 259-288.

Hinweis des Verlags Der Verlag bleibt in Hinblick auf geografische Zuordnungen und Gebietsbezeichnungen in veröffentlichten Karten und Institutsadressen neutral.

\section{Julia Gruevska}

Geschichte und Philosophie der Naturwissenschaften mit dem

Schwerpunkt Lebenswissenschaften mit Ernst-Haeckel-Haus

Friedrich-Schiller-Universität Jena

Berggasse 7

07745 Jena

Deutschland

Julia.Gruevska@uni-jena.de 